Wall ablation of heated compound-materials into non-equilibrium discharge plasmas

This content has been downloaded from IOPscience. Please scroll down to see the full text.

2017 J. Phys. D: Appl. Phys. 50074005

(http://iopscience.iop.org/0022-3727/50/7/074005)

View the table of contents for this issue, or go to the journal homepage for more

Download details:

IP Address: 146.175.11.111

This content was downloaded on 18/04/2017 at 08:45

Please note that terms and conditions apply.

You may also be interested in:

Theoretical investigation of the decay of an SF6 gas-blast arc using a two-temperature hydrodynamic model

WeiZong Wang, Joseph D Yan, MingZhe Rong et al.

Two-temperature thermodynamic and transport properties of SF6-Cu plasmas

Yi Wu, Zhexin Chen, Fei yang et al.

CO2 conversion in a gliding arc plasma: 1D cylindrical discharge model

Weizong Wang, Antonin Berthelot, Stanimir Kolev et al.

Non-equilibrium modelling of arc plasma torches

J P Trelles, J V R Heberlein and E Pfender

Pulsed arc discharge in dry air

Yasunori Tanaka, T Michishita and Y Uesugi

Thermal and electrical influences from bulk plasma in cathode heating modeling

Tang Chen, Cheng Wang, Xiao-Ning Zhang et al.

Computational non-chemically equilibrium model on the current zero simulation in a model N2 circuit breaker under the free recovery condition

Hao Sun, Yasunori Tanaka, Kentaro Tomita et al.

Energy equation formulations for two-temperature modelling of 'thermal' plasmas

P Freton, J J Gonzalez, Z Ranarijaona et al. 


\title{
Wall ablation of heated compound-materials into non-equilibrium discharge plasmas
}

\author{
Weizong Wang ${ }^{1,5,6,7}$, Linghan Kong ${ }^{1,2}$, Jinyue Geng ${ }^{3}$, Fuzhi Wei ${ }^{3}$ \\ and Guangqing $\mathrm{Xia}^{4}$
}

${ }^{1}$ Qian Xuesen Laboratory of Space Technology, China Academy of Space Technology, Beijing 100094, People's Republic of China

2 School of Aerospace, Tsinghua University, Beijing 100084, People's Republic of China

${ }^{3}$ Beijing Institute of Control Engineering, China Academy of Space Technology, Beijing 100094,

People's Republic of China

${ }^{4}$ State Key Laboratory of Structural Analysis for Industrial Equipment, School of Aeronautics and Astronautics, Dalian University of Technology, Dalian 116024, People's Republic of China

5 Current address: University of Antwerp, BE-2610 Wilrijk-Antwerp, Kingdom of Belgium

E-mail: wangweizong@gmail.com

Received 31 July 2016, revised 29 November 2016

Accepted for publication 29 December 2016

Published 24 January 2017

\begin{abstract}
The discharge properties of the plasma bulk flow near the surface of heated compound-materials strongly affects the kinetic layer parameters modeled and manifested in the Knudsen layer. This paper extends the widely used two-layer kinetic ablation model to the ablation controlled nonequilibrium discharge due to the fact that the local thermodynamic equilibrium (LTE) approximation is often violated as a result of the interaction between the plasma and solid walls. Modifications to the governing set of equations, to account for this effect, are derived and presented by assuming that the temperature of the electrons deviates from that of the heavy particles. The ablation characteristics of one typical material, polytetrafluoroethylene (PTFE) are calculated with this improved model. The internal degrees of freedom as well as the average particle mass and specific heat ratio of the polyatomic vapor, which strongly depends on the temperature, pressure and plasma non-equilibrium degree and plays a crucial role in the accurate determination of the ablation behavior by this model, are also taken into account. Our assessment showed the significance of including such modifications related to the non-equilibrium effect in the study of vaporization of heated compound materials in ablation controlled arcs. Additionally, a two-temperature magneto-hydrodynamic (MHD) model accounting for the thermal non-equilibrium occurring near the wall surface is developed and applied into an ablation-dominated discharge for an electro-thermal chemical launch device. Special attention is paid to the interaction between the non-equilibrium plasma and the solid propellant surface. Both the mass exchange process caused by the wall ablation and plasma species deposition as well as the associated momentum and energy exchange processes are taken into account. A detailed comparison of the results of the non-equilibrium model with those of an equilibrium model is presented. The non-equilibrium results show a non-equilibrium region near the plasma-wall interaction region and this indicates the need for the consideration of the influence of the possible departure from LTE in the plasma bulk on the determination of ablation rate.
\end{abstract}

Keywords: wall ablation, non-equilibrium plasma, two-temperature model, magnetohydrodynamic (MHD) model, capillary discharge

(Some figures may appear in colour only in the online journal)

\footnotetext{
${ }^{6}$ Author to whom any correspondence should be addressed.

${ }^{7}$ This article belongs to the special issue: Emerging Leaders, which features invited work from the best early-career researchers working within the scope of J. Phys. D. This project is part of the Journal of Physics series' 50th anniversary celebrations in 2017. Weizong Wang was selected by the Editorial Board of J. Phys. $D$ as an Leader. 


\section{Nomenclature}

\begin{tabular}{|c|c|}
\hline Symbol & Parameter \\
\hline$\Delta H$ & Ablation heat \\
\hline$\Gamma$ & Ablation/deposition rate \\
\hline$A_{\text {element }}$ & Area of the solid wall surface element \\
\hline$m_{i}(i=0,1,2)$ & $\begin{array}{l}\text { Average species mass at the boundaries }(0) \text {, } \\
(1) \text {, and (2) in figure } 1\end{array}$ \\
\hline$k$ & Boltzmann's constant \\
\hline$n C_{2}$ & Carbon particle density at the boundary (2) \\
\hline$P_{\mathrm{c}}$ & Characteristic pressure \\
\hline$T_{\mathrm{c}}$ & Characteristic temperature \\
\hline$\vec{J}$ & Current density vector \\
\hline$E_{\mathrm{d}}$ & Dissociation energy \\
\hline$\vec{E}$ & Electric field vector \\
\hline$\varphi$ & Electric potential \\
\hline$\sigma$ & Electrical conductivity \\
\hline$q_{\mathrm{e}}$ & $\begin{array}{l}\text { Electron enthalpy transported with the mass } \\
\text { flows }\end{array}$ \\
\hline$n_{\mathrm{e}}$ & Electron number density \\
\hline$T_{\mathrm{e}}$ & Electron temperature \\
\hline$T_{2, \mathrm{e}}$ & Electron temperature at the boundary (2) \\
\hline$k_{\mathrm{e}}$ & Electron thermal conductivity \\
\hline$e$ & Electronic charge \\
\hline$n_{2, \mathrm{e}}$ & Electrons number density at the boundary (2) \\
\hline$P_{\mathrm{v}}$ & Equilibrium vapor pressure \\
\hline $\operatorname{erfc}(\alpha)$ & Error function \\
\hline$V_{i}(i=1,2)$ & $\begin{array}{l}\text { Gas flow velocity at the boundaries (1) and } \\
\text { (2) in figure } 1\end{array}$ \\
\hline$T_{i}(i=0,1)$ & $\begin{array}{l}\text { Gas temperature at the boundaries (0) and (1) } \\
\text { in figure } 1\end{array}$ \\
\hline$g$ & Gravity coefficient \\
\hline$q(t)$ & Heat flux density \\
\hline$n_{2, \mathrm{~h}}$ & $\begin{array}{l}\text { Heavy particle number density at the } \\
\text { boundary (2) }\end{array}$ \\
\hline$T_{\mathrm{h}}$ & Heavy particle temperature \\
\hline$T_{2, \mathrm{~h}}$ & Heavy particle temperature at the boundary (2) \\
\hline$k_{\mathrm{h}}$ & Heavy particle thermal conductivity \\
\hline$q_{\mathrm{h}}$ & $\begin{array}{l}\text { Heavy particles enthalpy transported with the } \\
\text { mass flows }\end{array}$ \\
\hline$T_{s}$ & Initial room temperature \\
\hline$R_{\mathrm{a}}$ & Inner diameter of the capillary \\
\hline$Z$ & Internal partition function \\
\hline$E_{\mathrm{I}, r+1}$ & Ionization energy \\
\hline$\grave{o} E_{\mathrm{I}, r+1}$ & Lowering of the ionization energy \\
\hline$B_{i}(i=1,2)$ & Magnetic field magnitude at the boundaries \\
\hline
\end{tabular}

(1) and (2)

\section{$\overrightarrow{\boldsymbol{B}} \quad$ Magnetic flux density}

$\overrightarrow{\boldsymbol{A}} \quad$ Magnetic potential vector

$\rho_{i}(i=1,2) \quad$ Mass density at the boundaries (1) and (2)

$\overrightarrow{\boldsymbol{u}}$

$\varepsilon_{r}$

$\theta$

$h$

$Q_{\mathrm{J}}$

$Q_{\text {rad }}$

$Q_{\text {eh }}$
$P_{i}(i=0,1,2)$ Pressure at the boundaries (0), (1), and (2) in figure 1

$P_{\text {sonic }} \quad$ Pressure boundary with sonic speed

$\Delta P \quad$ Pressure correction resulted from the charged particles interactions

$\beta \quad$ Proportionality coefficient

$T_{\text {ex }} \quad$ Reaction excitation temperature

$\rho_{\mathrm{m}} \quad$ Solid wall mass density

$T_{\mathrm{m}} \quad$ Solid wall temperature

$u_{\text {sonic }} \quad$ Sonic speed

$z \quad$ Specie charge

$n_{i} \quad$ Species number density

$n_{i}(i=0,1,2) \quad$ Species number density at the boundaries (0), (1), and (2) in figure 1

$h_{\mathrm{e}} \quad$ Specific enthalpy of electrons

$h_{\mathrm{h}} \quad$ Specific enthalpy of heavy particles

$C_{p}, m \quad$ Specific heat of the solid wall

$\gamma \quad$ Specific heat ratio

$k_{\mathrm{m}} \quad$ Thermal conductivity of the solid wall

$q_{\mathrm{T}} \quad$ Total enthalpy transported with the mass flows

$\rho \quad$ Total mass density

$h_{\mathrm{T}} \quad$ Total specific enthalpy

$k_{\mathrm{T}} \quad$ Total thermal conductivity

$\mu \quad$ Vacuum permeability

$v_{\text {flux }} \quad$ Velocity by which the mass enters or escapes the plasma element

Viscous stress tensor

Volume mass generation or loss rate by ablation or deposition

$V_{\text {element }} \quad$ Volume of the plasma's element adjacent to the wall

$\xi_{\text {eh }} \quad$ Volumetric collision frequency between the electrons and the heavy particles

$T_{\text {wall } \quad \text { Wall surface temperature }}$

\section{Introduction}

Ablation-controlled plasmas are widely used in many technical applications such as circuit breakers [1-3], soft x-ray sources [4], pulsed plasma thrusters (PPT) [5-7], electrothermal-chemical (ETC) launch devices [8-10], and laser ablation devices $[11,12]$. The ablated compound-materials vaporize due to the strong heating effect by the dissipated electric energy of highly-energetic, high-intensity plasma discharge and generate a mass flux into the plasma. The ablated vapor participates in the discharge itself and significantly influences the plasma profile such as the temperature, the velocity and the pressure. Meanwhile, the carbon particles in the plasma tend to deposit on the wall surface, generating a reverse mass flux to the wall. Therefore, an accurate description of the wall ablation characteristic of heated compoundmaterials interacting with discharge plasma is of great interest in the improvement of the device's performance.

There has been much effort devoted to studying the mechanisms governing the plasma-wall interaction in ablation process [13-28]. Among these, a model based on the Langmuir law which assumes that in the layer adjacent to the wall 
surface, half the particles move into the plasma and the other half move back to the wall takes into account only the wall surface temperature to calculate the ablated mass [13]. This model, which assumes the boundary velocity at the ablation surface is equal to the sonic velocity, was applied to a PPT [7]. It is noted that this model is limited to the case of material ablation into a vacuum environment. However, in most cases, the ablated vapor does not expand into vacuum but rather into a volume discharge. Additionally, a model assuming that the ablation is caused by radiation energy is widely used in the simulation of switching arcs in circuit breakers [1, 19] and ETC plasma guns [15-18]. In this model, a non-dimensional transparency factor, which represents the fraction of the radiated energy that leads to wall ablation, is empirically chosen to match the experimental results. The remaining radiation can either be reabsorbed in the bulk plasma, cause heating of the wall material, or escape from the capillary. Recently, there have been many reports of studies based on the two-layer kinetic model [20-29]. This model developed by Keidar et al is based on the Anisimov's model [30, 31] for the laser ablation of metal to describe the wall-plasma transition region and the returned atom flux that forms in the non-equilibrium layer during the ablation is taken into account. The condition that the ablation surface velocity is lower than the sonic velocity in high-density discharge plasma is accounted for compared with the above mentioned Langmuir model. The ablation rate can be determined from the plasma bulk parameters (plasma density and pressure) under the assumption that the bulk plasma is under the local thermodynamic equilibrium (LTE) condition. Keidar et al's derivation [20] in the energy flux conservation only takes into account the contribution of the energy associated with the translational degrees of freedom and the derived expressions are valid only for a monatomic vapor gas. Assuming that Teflon vapor is composed of $\mathrm{CF}_{2}$ molecules and the internal degrees of freedom are zero in their work, the effect of the change of the average molecular mass as well as the specific heat ratio with temperature and pressure on the mass, momentum, and energy fluxes conservation is ignored as well.

Further improvement of this two-layer kinetic ablation model includes the consideration of the accessible internal degrees of freedom and temperature/pressure-dependent average species mass as well as the specific heat ratio $[25,27$, $28]$ in the application of conservation of mass, momentum, and energy fluxes through both the kinetic non-equilibrium Knudsen layer and the hydrodynamic layer. Among the three models describing the wall ablation process mentioned above, the kinetic model is an appropriate choice for predicting the ablation flux because its predictions agree well with the experimental results. Moreover, it does not need any empirically-determined parameters, and thus has broad applicability [32]. Based on the two-layer ablation kinetic model, a steady magneto-hydrodynamic (MHD) model of an ablationdominated capillary discharge is developed to simulate the distinct 2D features that exist in the distribution of the plasma properties [32]. In the above mentioned two-layer kinetic ablation model and the MHD model, it is assumed that at the boundary between the hydrodynamic equilibrium layer and the plasma bulk flow, all species (ions, neutrals and electrons) reach thermal equilibrium. However, in many cases, departure from thermal equilibrium occurs in near-wall regions due to the strong cooling effect by the cold wall [33]. To model the plasma in these applications more accurately, the LTE assumption needs to be abandoned in favor of a thermal nonequilibrium description where the heavy particles and electrons separately follow a Maxwell-Boltzmann distribution but at different temperatures. For this purpose, in the literature, a two-temperature MHD model has been developed in which the electron and the heavy species are treated as two different perfect gases [34-36]. However, the interaction between the wall and the non-equilibrium plasma bulk flow has been simplified and the wall ablation process has been neglected.

In order to contribute to a better understanding of the process of interaction between the wall surfaces and plasma arcs, this paper extends the widely used two-layer kinetic ablation model to the ablation controlled non-equilibrium discharge. Modifications to the governing set of equations, to account for this effect, are derived and presented by assuming that the temperature of the electrons deviates from that of the heavy particles in the plasma bulk flow. Based on the improved two-layer kinetic ablation model, for the first time, a two-temperature MHD model accounting for the thermal non-equilibrium occurring near the wall surface is developed and applied into an ablation-dominated discharge for an ETC launch device. Additionally, note that Li et al's steady model [32] has its limits because the competition of the ablation and deposition with time in one discharge pulse could not be observed. Moreover, the wall surface temperature was assumed to be constant $\left(T_{\text {wall }}=650 \mathrm{~K}\right)$ despite the application of different discharge currents values. The effect of the time-dependent wall surface temperature on the ablation rate and plasma parameters could not be fully investigated. Therefore, for the first time, our current paper performs a self-consistent calculation of the wall temperature distribution in a 2D geometry under both the steady and transient conditions.

To be more concrete, the paper is arranged as follows. In section 2, we first present the mathematical derivation of the two-layer kinetic ablation model in the ablation controlled non-equilibrium discharge where the electrons and heavy particles are characterized by two temperatures in chemical non-equilibrium. The needed data for the determination of the ablation process are calculated, and the quantitative relationships between the ablation rate and non-equilibrium plasma temperature and density of the ablated PTFE vapor are described in detail for one typical material, PTFE. The internal degrees of freedom as well as the average particle mass and specific heat ratio of the polyatomic vapor, which strongly depends on the temperature, pressure and non-equilibrium degree and plays a crucial role in the accurate determination of the ablation behavior by this model, are also taken into account. Section 3 introduces a two-temperature MHD model applied into an ablation-dominated discharge for an ETC launch device. The thermodynamic properties and transport coefficients as well as the data banks of the energy exchange term, which provide a prerequisite for reliable numerical simulation of plasmas' behavior, are computed. Special attention is paid to the interaction between the non-equilibrium 
plasma and the solid propellant surface under both the steady and transient conditions. The influence of departures from thermal equilibrium on the capillary discharge is discussed by comparing it with the results obtained using the LTE model if thermal equilibrium is assumed. Finally, conclusions are given in section 4 .

\section{Analysis of the ablation characteristics of PTFE into non-equilibrium discharge plasmas}

\subsection{Mathematical models}

The wall-plasma interaction occurs in a non-equilibrium boundary layer between the plasma bulk and ablation surface. A two-layer kinetic model by Keidar et al [20] was developed to study the ablation characteristics in this region. This boundary layer includes a kinetic non-equilibrium Knudsen layer adjacent to the wall surface with a thickness of a few mean free paths and a collision-dominated non-equilibrium hydrodynamic layer with different electron and heavy particle temperatures. The Knudsen layer is used to describe the transition layer between the ablated wall (0) and fluid (1) in the ablation process. The non-equilibrium layer is used to describe the plasma process in the plasma sheath. Net ablation flux including the contribution of both the wall ablation and vapor deposition effuses from the outer boundary of the Knudsen layer into the non-equilibrium hydrodynamic layer. The quasi-neutral plasma bulk flow is beyond this layer. Previous works [20-29] assume all species (ions, neutrals, and electrons) to reach thermal equilibrium for which the electrons and heavy particles have an identical temperature. In our current work, we take into account the possible departure from thermal equilibrium in the plasma bulk near the surface due to the rapid cooling effect by the cold wall. The non-equilibrium plasma bulk flow is characterized by two different temperatures for electrons and heavy particles $\left(T_{\mathrm{e}}\right.$ and $\left.T_{\mathrm{h}}\right)$ but the same bulk flow velocity $\left(V_{2}\right)$ because of frequent collisions between electrons and heavy particles.

The structure of the non-equilibrium layer is presented in figure 1. By applying conservation laws of mass, momentum and translational energy across the Knudsen layer, Keidar et al [20] derive the relationship between the parameters at the solid surface (heavy species number density $n_{0}$, surface temperature $T_{0}$ ) and the outer boundary of the Knudsen layer (heavy species number density $n_{1}$, temperature $T_{1}$ and velocity $\left.V_{1}\right)$. In their work, they assume the species at the boundaries (0) and (1) are all atomic species with the same mass $\left(m_{0}=m_{1}\right)$. However, not only the atoms but also the molecules exist at these boundaries and their average particle mass may be different. For this, taking into account the accessible internal degrees of freedom and the different average particle mass at the boundaries of the Knudsen layer, Zaghloul gives the following relationships of the heavy particle parameters [25].

$$
\frac{m_{0} n_{0}}{2 \sqrt{\pi d_{0}}}=m_{1} n_{1} V_{1}+\beta \frac{m_{1} n_{1}}{2 \sqrt{\pi d_{1}}}\left[\exp \left(-\alpha^{2}\right)-\alpha \sqrt{\pi} \operatorname{erfc}(\alpha)\right]
$$

$\frac{m_{0} n_{0}}{4 d_{0}}=\frac{m_{1} n_{1}}{2 d_{1}}\left\{\left(1+2 \alpha^{2}\right)-\beta\left[\left(0.5+\alpha^{2}\right) \operatorname{erfc}(\alpha)-\frac{\alpha \exp \left(-\alpha^{2}\right)}{\sqrt{\pi}}\right]\right\}$

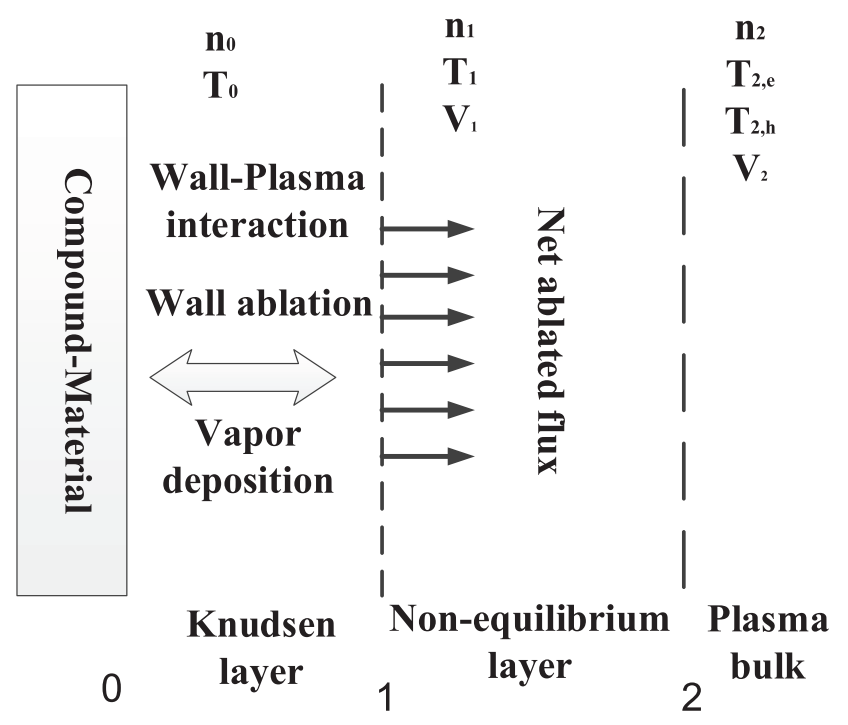

Figure 1. Schematic illustration of the layer structure near the ablated surface.

$$
\begin{aligned}
& \frac{m_{0} n_{0}}{\left(\pi d_{0}\right)^{1.5}} \frac{\gamma+1}{4(\gamma-1)} \frac{\pi d_{1}^{1.5}}{m_{1} n_{1}}=\alpha\left(\alpha^{2}+\frac{\gamma}{\gamma-1}\right) \\
& \quad-\frac{\beta}{2}\left[\alpha\left(\alpha^{2}+\frac{\gamma}{\gamma-1}\right) \operatorname{erfc}(\alpha)-\left(\alpha^{2}+\frac{\gamma+1}{2(\gamma-1)}\right) \frac{\exp \left(-\alpha^{2}\right)}{\sqrt{\pi}}\right]
\end{aligned}
$$

where $T, n, V$, and $m$ are, respectively, temperature, number density, velocity and average particle mass of the plasma, with subscripts 0,1 , and 2 denoting the values at boundaries $(0),(1)$, and (2) in figure 1. $\gamma$ is the specific heat ratio in the Knudsen layer. $d_{0}=m_{0} / 2 k T_{0}, d_{1}=m_{1} / 2 k T_{1}, \alpha=V_{1} / \sqrt{2 k T_{1} m_{1}}$, $\operatorname{erfc}(\alpha)$ is the error function and $\beta$ is a proportionality coefficient representing the quantity of back flow particles.

Following the treatment of Keidar et al [20], we apply mass and momentum conservation equations for heavy particles in the hydrodynamic layer (assumed as a single fluid model) between boundaries (1) and (2) and give the following relations:

$$
\begin{aligned}
& m_{1} n_{1} V_{1}=m_{2} n_{2} V_{2} \\
& n_{1} k T_{1}+m_{1} n_{1} V_{1}^{2}+B_{1}^{2} /(2 \mu)=n_{2, \mathrm{e}} k T_{2, \mathrm{e}}+n_{2, \mathrm{~h}} k T_{2, \mathrm{~h}}+m_{2} n_{2} V_{2}^{2} \\
&+B_{2}^{2} /(2 \mu)
\end{aligned}
$$

where $V_{2}, n_{2}, T_{2, \mathrm{~h}}$ and $T_{2, \mathrm{e}}$ are the velocity, the number density, heavy particle temperature and electron temperature at the outer boundary of the hydrodynamic layer, respectively; $m_{1}, B_{1}, m_{2}, B_{2}$ are the molecular mass and magnetic field intensity at the boundaries (1) and (2), respectively; $n_{2, \mathrm{~h}}$ and $n_{2, \mathrm{e}}$ are the number density of heavy particles and electrons. The total species number density at the boundary (2) $n_{2}=n_{2, \mathrm{~h}}+n_{2, \mathrm{e}} \cdot \mu$ and $k$ are vacuum permeability and the Boltzmann constant.

The terms related to the magnetic field intensity in formulas (4) and (5) reflect the contribution of the electromagnetic field on the plasma acceleration, for example in the case of PPT. For the ablative capillary discharge in our work, plasma 
aerodynamic acceleration is dominant $\left(B_{1}=0 ; B_{2}=0\right)$. In this case, formulas (4) and (5) yield the expression of $V_{1}$

$\frac{V_{1}^{2}}{2 k T_{1} m_{1}}=\alpha^{2}=\frac{1}{2} \frac{\left(\frac{n_{2, \mathrm{e}} T_{2, \mathrm{e}}+n_{2, \mathrm{~h}} T_{2, \mathrm{~h}}}{n_{1} T_{1}}-1\right)}{\left(1-\frac{m_{1} n_{1}}{m_{2} n_{2}}\right)}=\frac{1}{2} \frac{\left(\frac{P_{2}}{P_{1}}-1\right)}{\left(1-\frac{\rho_{1}}{\rho_{2}}\right)}$

where $P_{2}=n_{2, \mathrm{e}} k T_{2, \mathrm{e}}+n_{2, \mathrm{~h}} k T_{2, \mathrm{~h}}$ and $P_{1}=n_{1} k T_{1}$ are the pressure and $\rho_{1}$ and $\rho_{2}$ are the mass density at the boundaries 1 and 2 , respectively.

By specifying that the pressure at the wall surface $P_{0}=n_{0} k T_{0}$ is equal to the equilibrium vapor pressure of the material being ablated at temperature $T_{0}$, the system of equations is closed.

$$
P_{0}=P_{\mathrm{v}}=P_{\mathrm{c}} \exp \left(-T_{\mathrm{c}} / T_{0}\right)
$$

where $P_{\mathrm{v}}$ is the equilibrium vapor pressure, $P_{\mathrm{c}}=1.84 \times 10^{15}$ $\mathrm{Nm}^{-2}$ and $T_{\mathrm{c}}=20815 \mathrm{~K}$ are the characteristic pressure and temperature, respectively [33].

The solution of the problem depends upon the plasma mass density $\rho_{2}$, plasma pressure $P_{2}$ and surface temperature $T_{0}$. The parameter $\rho_{2}$ and plasma pressure $P_{2}$ are determined by the bulk plasma flow. Provided with values of $\rho_{2}, P_{2}$, and $T_{0}$, the equation sets can be solved and the ablation rate can be calculated as

$$
\Gamma=m_{1} n_{1} V_{1}
$$

\subsection{Species composition of two-temperature plasma}

The non-equilibrium characteristics in the plasma bulk flow near the wall surface is described by a two-temperature model which includes the assumption of local chemical equilibrium (LCE) in order to take into account the thermal departure from equilibrium, the two temperatures being the electron temperature $\left(T_{\mathrm{e}}\right)$ and the heavy particle temperature $\left(T_{\mathrm{h}}\right)$. Since the mass difference between the heavy particles is minor compared to that between electrons and heavy particles, all the heavy particles have the same temperature $T_{\mathrm{h}}$, which is sometimes called the plasma temperature or gas temperature. The plasma species composition, which is a function of the pressure, electron temperature and heavy particle temperature, is determined by the mass action law, the quasi-neutrality condition and Dalton's law of partial pressures [37, 38]. An important deviation from the ideal gas at the pressures considered here is the effective shielding of charged species by species of the opposite charge. The Debye-Hückel correction, which represents the additional effect of electrostatic interactions between the charged particles, is taken into account in determining the two-temperature plasma composition. This requires the consideration of the lowering of the ionization potential and correction terms to be added to Dalton's Law, i.e. the equation of state is described as follows.

For an ablated PTFE plasma, the plasma is primarily composed of 24 species, namely, $\mathrm{CF}_{4}, \mathrm{CF}_{3}, \mathrm{CF}_{2}, \mathrm{CF}, \mathrm{C}_{2} \mathrm{~F}_{4}, \mathrm{C}_{2} \mathrm{~F}_{2}$, $\mathrm{C}_{5}, \mathrm{C}_{4}, \mathrm{C}_{3}, \mathrm{C}_{2}, \mathrm{~F}_{2}, \mathrm{~F}, \mathrm{C}, \mathrm{CF}_{3}^{+}, \mathrm{CF}_{2}^{+}, \mathrm{CF}^{+}, \mathrm{F}^{+}, \mathrm{C}^{+}, \mathrm{F}^{-}, \mathrm{C}^{2+}, \mathrm{F}^{2+}$, $\mathrm{C}^{3+}, \mathrm{C}^{3+}$, and $e$ for the temperatures considered here. The calculations including more species show that other species are neglected because of their low amount appearing in the system.

In this case, the Guldberg-Waage equation and generalized Saha law are written using van de Sanden et al's derivation [39] for the dissociation reaction $a b \rightarrow a+b$ and the ionization reaction $a^{r+} \rightarrow a^{(r+1)+}+e$, respectively.

$\frac{n_{a} n_{b}}{n_{a b}}=\frac{Z_{a} Z_{b}}{Z_{a b}}\left[\frac{2 \pi k T_{\mathrm{h}}}{h^{2}}\right]^{3 / 2}\left[\frac{m_{a} m_{b}}{m_{a b}}\right]^{3 / 2} \exp \left[-\frac{E_{\mathrm{d}}}{k T_{\mathrm{ex}}}\right]$

$n_{\mathrm{e}}\left[\frac{n_{r+1}}{n_{r}}\right]=2\left[\frac{Z_{r+1}}{Z_{r}}\right]\left[\frac{2 m_{\mathrm{e}} \pi k T_{\mathrm{e}}}{h^{2}}\right]^{3 / 2} \exp \left[-\frac{E_{\mathrm{I}, r+1}-\delta E_{\mathrm{I}, r+1}}{k T_{\mathrm{ex}}}\right]$

where $Z, E_{\mathrm{I}, r+1}$ and $E_{\mathrm{d}}$ are, the generalized internal partition function, the ionization energy and dissociation energy, respectively; $h, \grave{o} E_{\mathrm{I}, r+1}$ are the Planck's constant and the lowering of the ionization energy. The lowering of the ionization energy, which limits the number of energy levels that should be considered, particularly at higher pressures, is taken into account using the approach of Wang et al [38] for two-temperature plasma. Both the contributions of electrons and ions are included in the determination of the effective shielding distance of charged particles. This is an important correction for pressures above $10 \mathrm{~atm} . n$ and $m$ are the specie number density and mass. The subscript $r$ indicates $r$-times ionized species or molecules. $a b, a$ and $b$ denote the reactant and the two products of the dissociation chemical reactions, respectively, and $T_{\mathrm{ex}}$ is the reaction excitation temperature. Here we use the heavy particle temperature $T_{\mathrm{h}}$ as an excitation temperature for the dissociation reaction and the electron temperature $T_{\mathrm{e}}$ as an excitation temperature for the ionization reaction.

Dalton's law of partial pressures is stated as

$$
P=\sum_{i \neq \mathrm{e}} n_{i} k T_{\mathrm{h}}+n_{\mathrm{e}} k T_{\mathrm{e}}+\Delta P
$$

where $\Delta P$ is the pressure correction that results from the charged particles interactions, detailed in our previous work of [38].

The quasi-neutrality condition reads as

$$
\sum_{s} n_{s} z_{s}=0
$$

where $z_{s}$ is the charge number of species $s$. Equations (9)-(12) allow for the calculation of the plasma composition for a given total pressure and temperature.

Species compositions for plasmas of ablated PTFE vapor as a function of the temperature of the electrons under different non-equilibrium degrees are shown in figures 2 and 3 . In the case of thermal equilibrium the electron temperature is equal to the heavy particle temperature, and the results are indicated for the temperature range in which significant amounts of molecular species are present, i.e. up to $8000 \mathrm{~K}$. At room temperature, the dominant ablation products are the $\mathrm{CF}_{4}$ and $\mathrm{C}_{5}$ species. $\mathrm{CF}_{4}$ further dissociates into $\mathrm{CF}_{3}$ and $\mathrm{CF}_{2}$ at around $2300 \mathrm{~K}$ and $\mathrm{C}_{5}$ dissociates into $\mathrm{C}_{4}$ and $\mathrm{C}_{3}$ at around $2800 \mathrm{~K}$. Further temperature increase brings the dissociation of $\mathrm{CF}_{2}$ into $\mathrm{CF}$ and $\mathrm{F}$ at around $3400 \mathrm{~K}$. The decomposition of $\mathrm{CF}$ into $\mathrm{C}$ and $\mathrm{F}$ atoms and $\mathrm{C}_{3}$ into $\mathrm{C}_{2}$ and $\mathrm{C}$ atoms occurs at around $5000 \mathrm{~K}$. The first ionization 

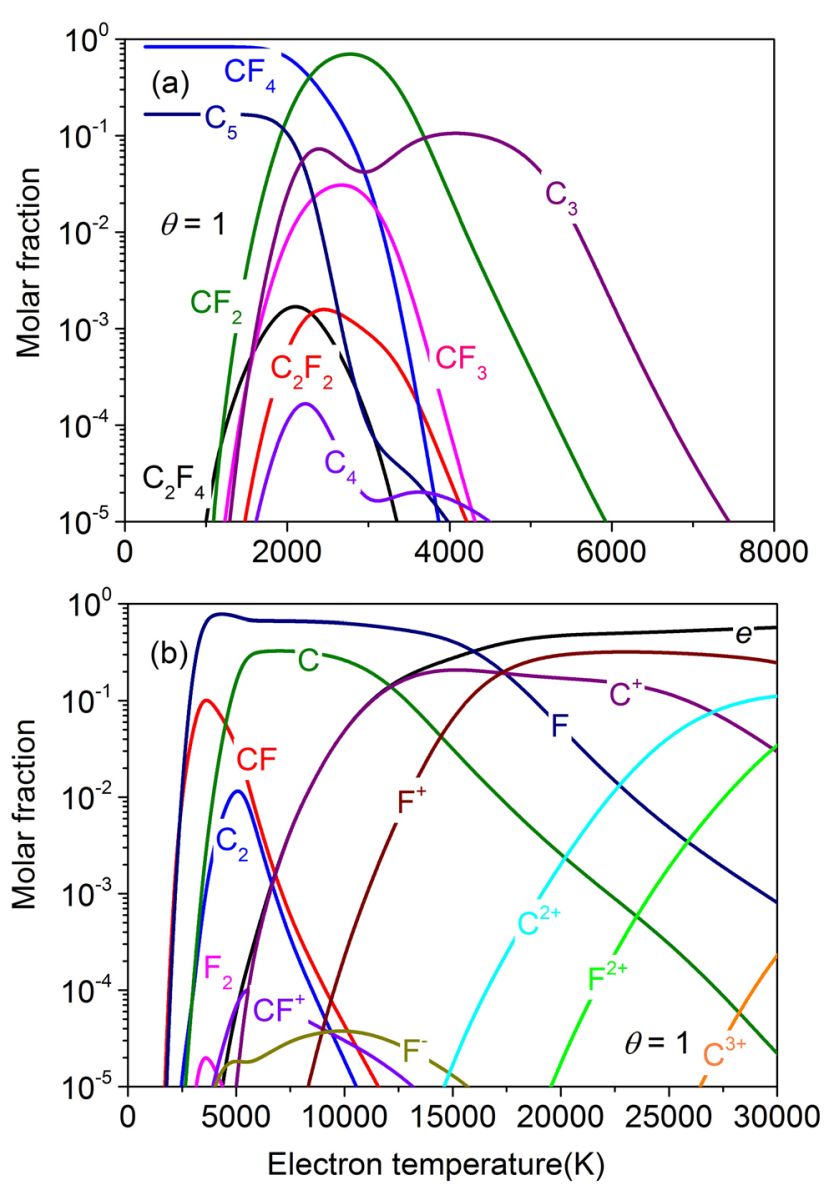

Figure 2. Temperature dependence of the molar fraction of polyatomic species (a) as well as diatomic species and monatomic (b) in equilibrium plasmas of ablated PTFE vapor at atmospheric pressure.

of $\mathrm{C}$ and $\mathrm{F}$ occurs at around the temperature of $10000 \mathrm{~K}$ and $17500 \mathrm{~K}$, respectively. The atomic carbon ionization takes place at a relatively lower temperature due to its ionization potential $(11.2 \mathrm{eV})$ which is lower than the fluorine atoms $(17.4 \mathrm{eV})$. Similar trends are found for their second and third ionizations which occur at much higher temperature ranges.

Under the non-thermal equilibrium condition, the dissociation reactions which are governed by the heavy particle temperature as described in equation (9) $T_{\mathrm{ex}}=T_{\mathrm{h}}$ are shifted towards higher electron temperature as presented in figure 3 for the non-equilibrium degree of $\theta=2$ which is defined as the ratio of electron temperature to heavy particle temperature. The ionization of neutral atoms is controlled by the electron temperature. This means once $\mathrm{CF}$ and $\mathrm{C}_{3}$ are dissociated into $\mathrm{C}$ and $\mathrm{F}$ atoms and $\mathrm{C}_{2}$ and $\mathrm{C}$ atoms and the ionization of atomic species can take place in a short amount of time because the temperature of the electrons has already reached a high value even though the temperature of the heavy particles is still relatively lower. As indicated in figures 2 and 3, one can see that at a given pressure, the species composition is not only affected by the electron temperature but also that of the heavy particle. Hence, the departure from the equilibrium condition will influence the plasma species composition which further changes the plasma parameters and hence the chararcteristics of the wall ablation. This will be discussed in the following section.
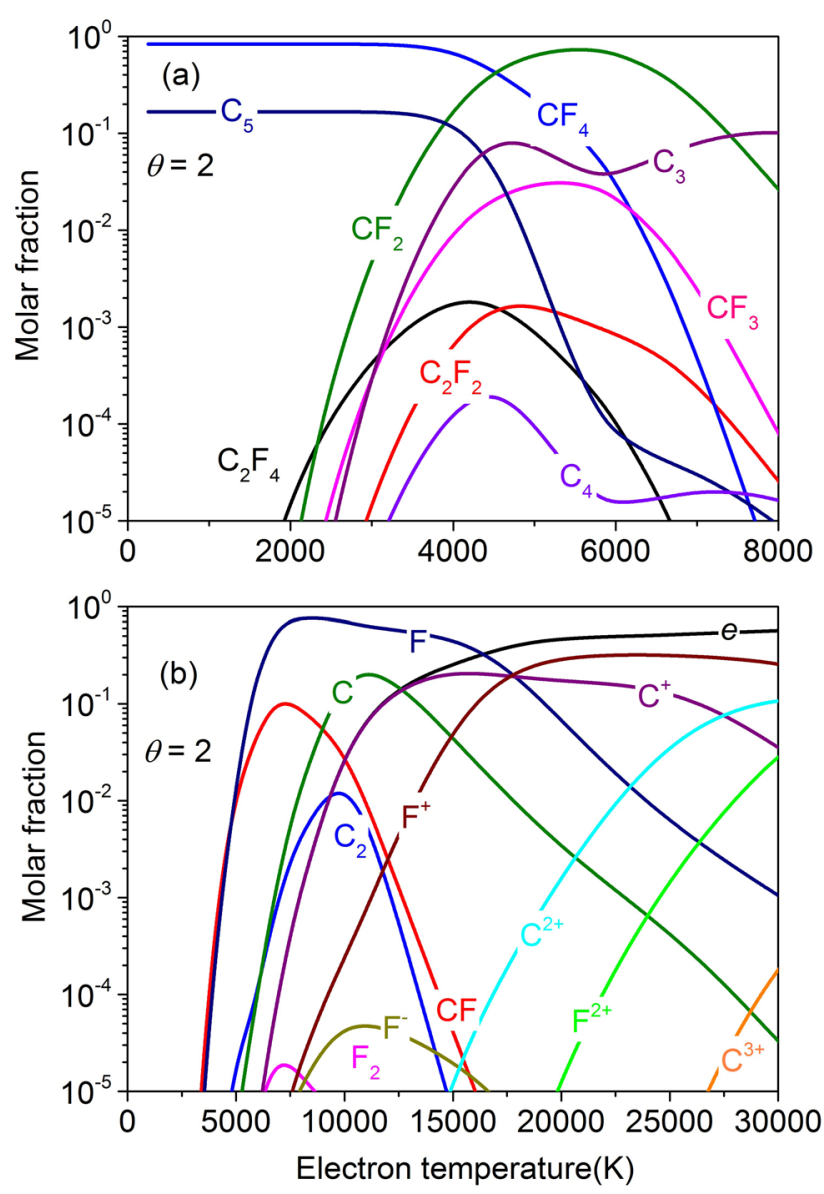

Figure 3. Temperature dependence of the molar fraction of polyatomic species (a) as well as diatomic species and monatomic species (b) in non-equilibrium plasmas of ablated PTFE vapor under the non-equilibrium degree of $\theta=2$ at atmospheric pressure.

Li et al [27] show that when the plasma bulk flow is under equilibrium, the average particle mass and specific heat ratio which strongly depend on the temperature and pressure can influence the wall ablation behavior. Therefore, in this work, during the iterative solution of the system of equations (1)-(8), the average particle mass and specific heat ratio when a single $f$ luid model is assumed, are determined as functions of both temperature and pressure at the boundaries (0) and (1). Additionally, as discussed above, departure from equilibrium in the plasma bulk flow can affect the plasma species composition $n_{2}$. Furthermore, it modifies the physical parameters of equation (6), i.e. the plasma mass density $\rho_{2}$, the specific heat ratio $\gamma$ as well as the average particle mass $m_{2}$. Therefore, the dependence of these plasma properties on the non-equilibrium degree together with the plasma pressure and temperature are taken into account as well. For example, as indicated in figures 4 and 5 , it is apparent that the plasma mass density as well as the average particle mass depend strongly on the nonequilibrium degree at the same pressure. At a given electron temperature, a larger plasma non-equilibrium degree leads to a higher plasma mass density and average particle mass. It has been verified that the effect of the average particle mass (the mass density) variation on the ablation process is critical [25, 28]. As a result, the influence of the non-equilibrium degree, 


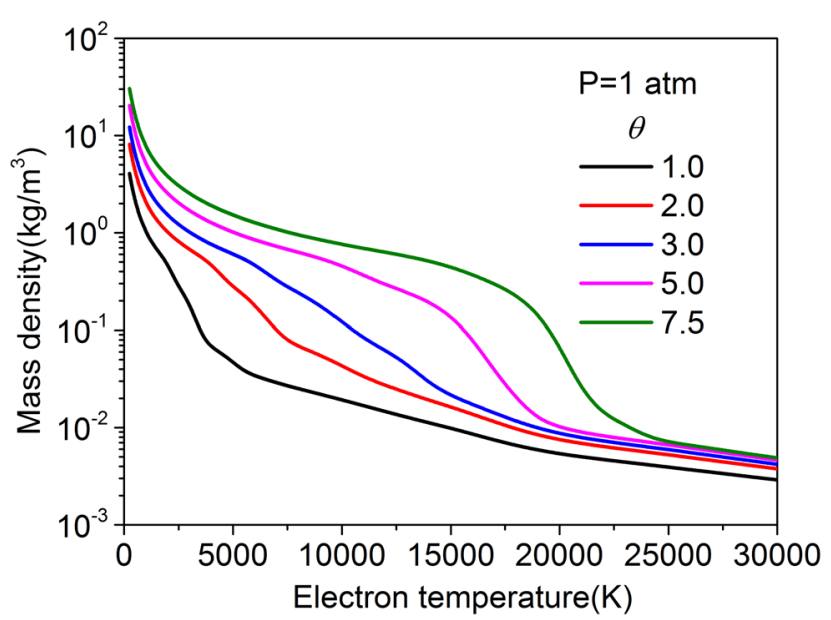

Figure 4. Mass density of equilibrium and non-equilibrium plasmas of ablated PTFE vapor under different non-equilibrium degrees at atmospheric pressures.

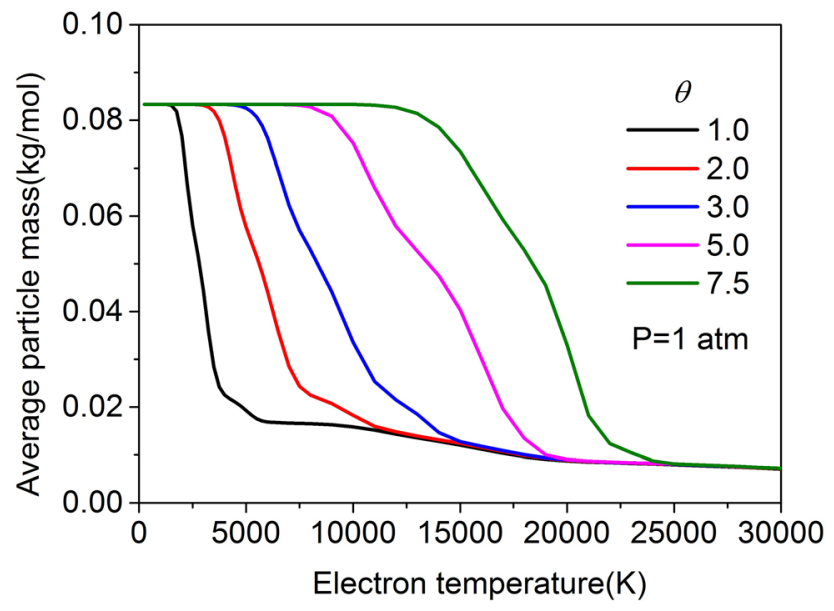

Figure 5. Average particle mass of equilibrium and nonequilibrium plasmas of ablated PTFE vapor under different non-equilibrium degrees.

which can change the average particle mass as well as the plasma mass density, is also significant. This will be further discussed in the following section.

\subsection{Calculated results of ablation data}

Based on the aforementioned model, as presented in figures 6 and 7 , we calculate the velocity $V_{1}$ and the ablation rates for PTFE as a function of the electron temperature under both the equilibrium and non-equilibrium conditions flow for different PTFE surface temperatures. A total species number density $n_{2}$ equal to $1.0 \times 10^{24} \mathrm{~m}^{-3}$ is used in the calculation. As we can see, both the velocity $V_{1}$ and the ablation rates are very sensitive to the temperature of the electrons at a given surface temperature and plasma density and decrease with increasing electron temperature. In the entire temperature range of the electrons considered, the velocity $V_{1}$ and the ablation rates have a larger value under the non-equilibrium condition. Moreover, the values of velocity $V_{1}$ and the ablation rates under the nonequilibrium condition reaches zero value (the critical condition $\alpha=0$ and $P_{2}=P_{1}=P_{0}$ for obtaining a physically acceptable

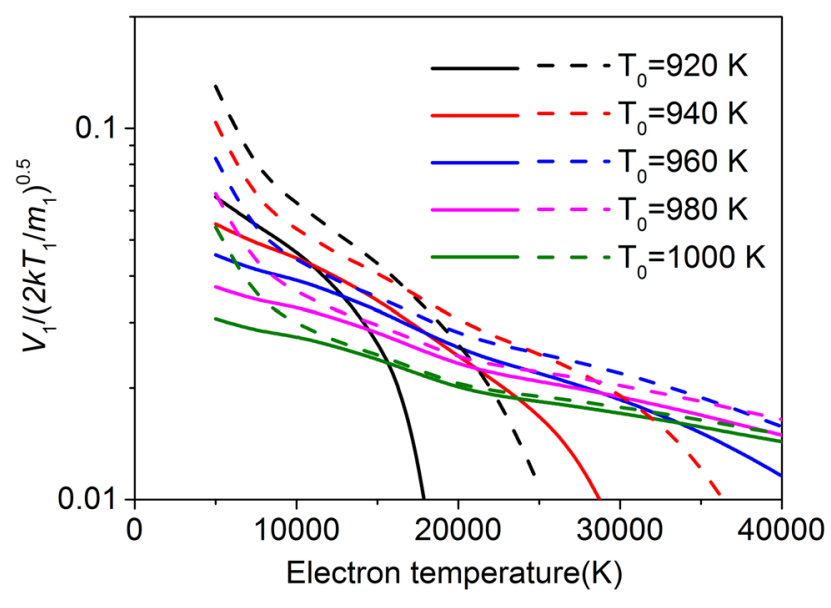

Figure 6. The velocity $V_{1}$ as a function of the electron temperature of the plasma bulk flow with the PTFE surface temperature as a parameter. Solid line: equilibrium condition, dashed line: nonequilibrium degree $\theta=2$. The total species number density $n_{2}$ is equal to $1.0 \times 10^{24} \mathrm{~m}^{-3}$.

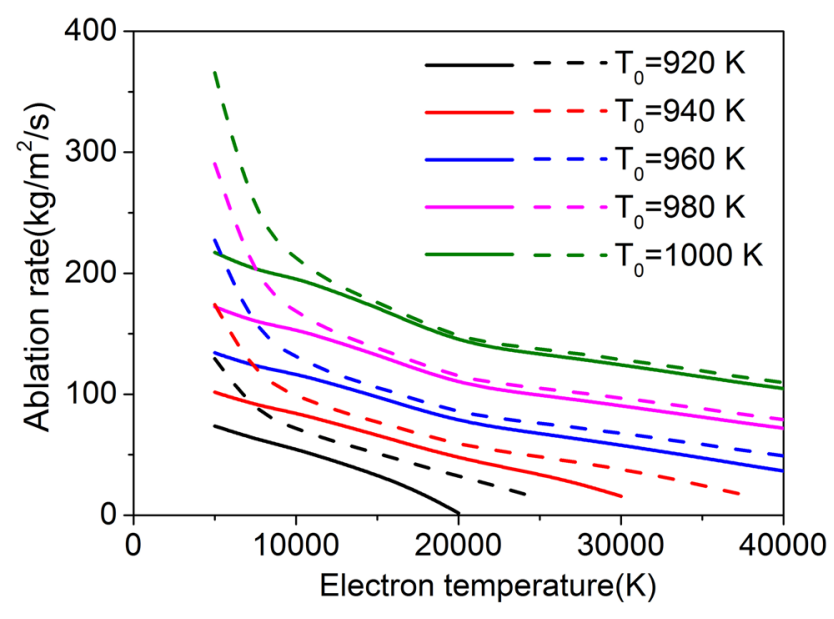

Figure 7. The ablation rate as a function of the electron temperature for different PTFE surface temperatures. Solid line: equilibrium condition, dashed line: non-equilibrium degree $\theta=2$. The total species number density $n_{2}$ is equal to $1.0 \times 10^{24} \mathrm{~m}^{-3}$.

solution of the system of equations (1)-(8) at a larger electron temperature compared with those under the equilibrium condition. This is attributed to that, for the former, according to Dalton's law of partial pressures (equation (11)), a smaller value of pressure is reached at a fixed total species number density and electron temperature as a result of a lower heavy particle temperature. This means that at a given total species number density, a higher electron temperature is needed to make the pressure reach the critical value $\left(P_{2}=P_{1}=P_{0}\right)$ and hence the zero values of velocity $V_{1}$ and ablation rate reaches zero value.

In order to investigate the influence of the pressure on ablation rates, figure 8 presents the contours of the ablation rate under both the equilibrium and non-equilibrium conditions in the $T_{0}-P_{2}$ plane for PTFE, for plasma temperature $T_{2}=10000 \mathrm{~K}$ and $20000 \mathrm{~K}$, which are typical of an ablation controlled plasma. The empty region beyond the zero line/ isosurface indicates that no ablation takes place for the corresponding plasma parameters. From the calculated ablation rates, one can see that the pressure can exert a large influence 

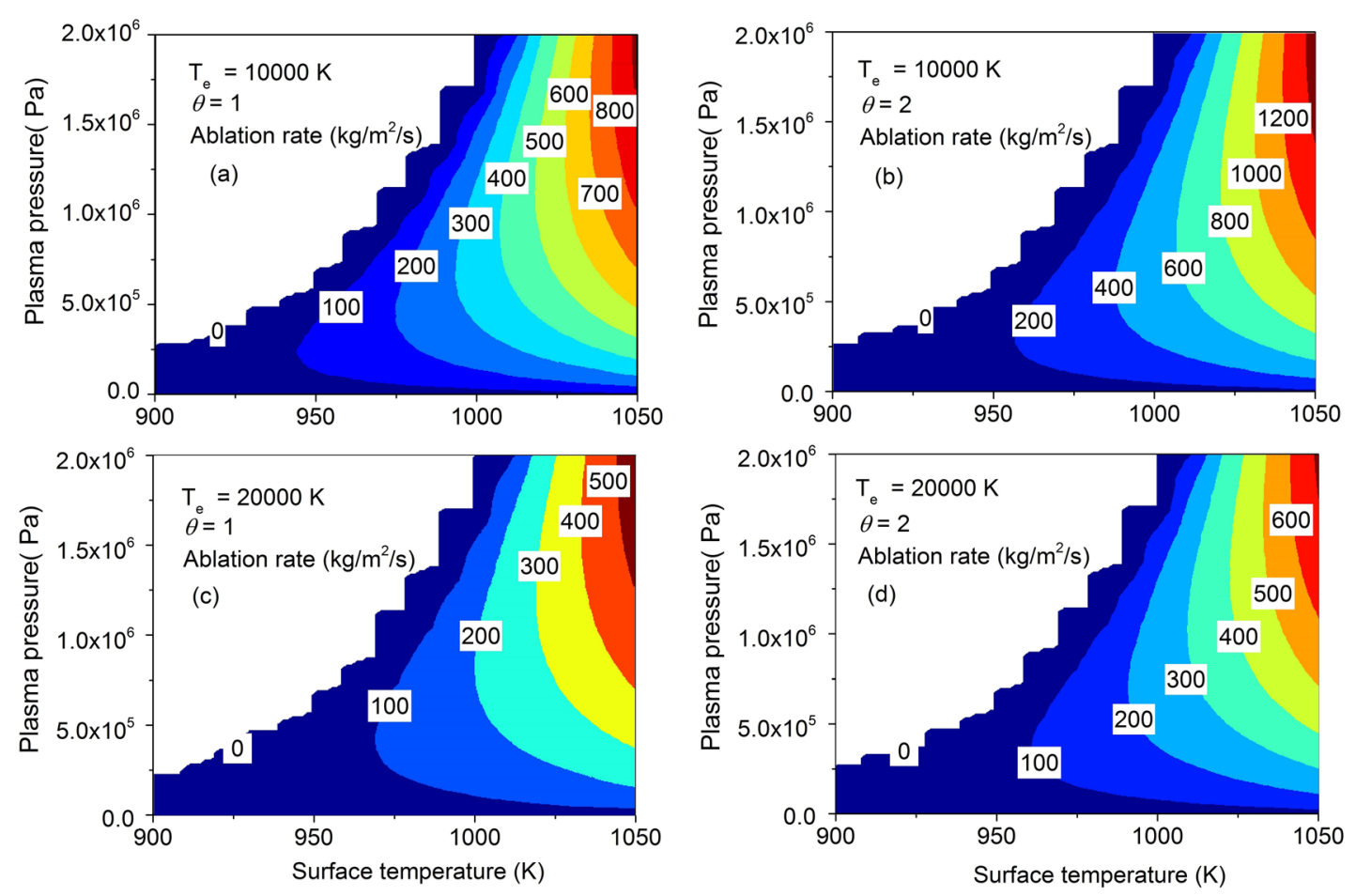

Figure 8. Comparison of ablation data for PTFE. In each plot, isolines of the ablation rate of PTFE are shown. (a) and (c) equilibrium condition; (b) and (d) non-equilibrium degree $\theta=2$.

on the ablation rate because the pressure can change the mass density apparently. The latter can further affect the ablation rate (see equation (6)).

Comparing the results of figures 8(a)-(d), one can also clearly see the influence of the departure from equilibrium on the computation of the ablation rate. At a given wall surface temperature, along the direction of the $y$-axis, most of the contours under the non-equilibrium condition is relatively more constricted than those under the equilibrium condition, indicating a more rapid change of ablation rate with pressure. As a result, the optimum plasma pressure at which the maximum ablation rate is obtained is shifted to a lower value. A similar tendency is also found for the change in ablation rate with surface temperature at a fixed pressure under the non-equilibrium condition. These trends show that at a given electron temperature, the change in ablation rate becomes more sensitive to the change in surface temperature and plasma pressure when departure from equilibrium takes place. Moreover, it demonstrates that a larger peak value of ablation rate is reached at a given surface temperature and electron temperature if the non-equilibrium effect is taken into account.

\section{Two-temperature MHD model of an ETC launch device}

\subsection{Assumptions}

The following assumptions and simplifications are adopted:

a. The plasma flow in the capillary is laminar continuum fluid.

b. The quasi-neutrality condition holds.

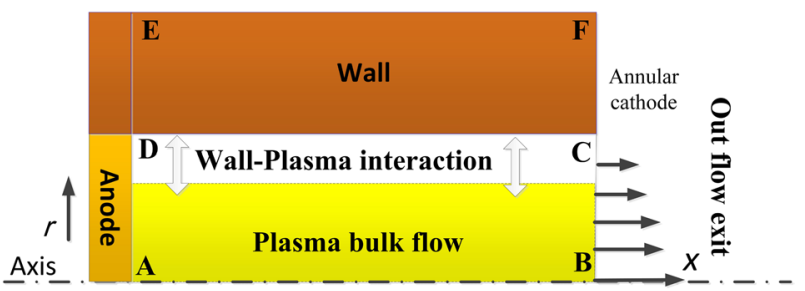

Figure 9. Schematic illustration of the capillary model.

c. Because of the frequent collisions between electrons and heavy particles, all plasma species are assured to attain the same velocity and only one momentum conservation equation is established.

d. The plasma is in a state of LCE. Heavy particles and electrons separately follow a Maxwell-Boltzmann distribution but are characterized by different temperatures. Therefore, energy conservation for electrons and heavy species needs to be treated separately to give different electron and heavy-species temperatures.

e. Anodic and cathodic sheaths have been neglected. The interaction between the electrodes and the arc is not considered in the current study.

f. The plasma composition is $100 \%$ ablated PTFE vapor. The influence of the pre-filled air and the metal vapor from the fuse wire and electrode erosion is negligible [32].

\subsection{Computational domain and governing equations}

The geometry in our simulation of capillary discharge is the same as the one in Li et al's experiment [40] and Li et al's simulation [32], with a length of $26 \mathrm{~mm}$ and an inner diameter 
of $R_{a}=3.2 \mathrm{~mm}$. Unlike Li et al who considered a polyethylene (PE) solid wall, we take into account a capillary discharge in an environment of the ablated vapor of the PTFE wall. In figure 9, $\mathrm{AB}$ is the central axis. $\mathrm{AD}$ is the anode; $\mathrm{CD}$ is the boundary of the plasma bulk flow. BC is the vent. In the real configuration, there is an annular cathode around the vent. Here, we apply a treatment similar to that of Li et al's simulation [32] and the cathode is ignored in order to simplify the problem. A solid wall area with an outer radius of $10 \mathrm{~mm}$ is included in the calculation area and its temperature distribution is taken into account. Therefore, the calculation domain is divided into two regions: the arc column region and the solid wall region. The regions are treated separately as described in the following subsections.

3.2.1. Arc column and transition regions. The arc model is based on MHD theory and described by the usual NavierStokes equations except for the associated source terms which are duly modified by the electromagnetic effects. Different from Li et al's simulation, both the steady and time dependent transient conditions are taken into account. For completeness and for ease of discussion, the governing equations of the twotemperature model for an axisymmetric arc used here are presented as follows.

(1) Mass conservation equation

$$
\frac{\partial \rho}{\partial t}+\nabla \cdot(\rho \overrightarrow{\boldsymbol{u}})=\dot{\rho}
$$

where $\overrightarrow{\boldsymbol{u}}$ is the mass-averaged velocity of the plasma species, and $\rho$ is the total mass density. $\dot{\rho}$ is the volume mass generation or loss rate by ablation or deposition in unit $\left(\mathrm{kg} \mathrm{m}^{-3}\right)$.

(2) Momentum conservation equation

$$
\frac{\partial \rho \overrightarrow{\boldsymbol{u}}}{\partial t}+\nabla \cdot(\rho \overrightarrow{\boldsymbol{u}} \overrightarrow{\boldsymbol{u}})=-\nabla p+\nabla \cdot \overline{\bar{\tau}}+\overrightarrow{\boldsymbol{J}} \times \overrightarrow{\boldsymbol{B}}+\rho \overrightarrow{\boldsymbol{g}}+v_{\text {flux }} \dot{\rho}
$$

where $p$ is the total pressure, $\overline{\bar{\tau}}$ is the viscous stress tensor, $\overrightarrow{\boldsymbol{J}} \times \overrightarrow{\boldsymbol{B}}$ is the Lorentz force, $\overrightarrow{\boldsymbol{J}}$ being the current density and $\overrightarrow{\boldsymbol{B}}$ the magnetic flux density induced by the current, and $g$ is the gravity coefficient.

\section{(3) Energy conservation equation}

The energy conservation equations for electrons and heavy particles are, respectively

$$
\begin{gathered}
\frac{\partial \rho h_{\mathrm{e}}}{\partial t}+\nabla \cdot\left(\rho h_{\mathrm{e}} \overrightarrow{\boldsymbol{u}}\right)=\nabla \cdot\left(k_{\mathrm{e}} \nabla T_{\mathrm{e}}\right)-Q_{\mathrm{eh}}-Q_{\mathrm{rad}}+Q_{\mathrm{J}} \\
\quad+\frac{5 k}{2 e} \overrightarrow{\boldsymbol{J}} \cdot \nabla T_{\mathrm{e}}+q_{\mathrm{e}} \dot{\rho} \\
\frac{\partial \rho h_{\mathrm{h}}}{\partial t}+\nabla \cdot\left(\rho h_{\mathrm{h}} \overrightarrow{\boldsymbol{u}}\right)=\nabla \cdot\left(k_{\mathrm{h}} \nabla T_{\mathrm{h}}\right)+Q_{\mathrm{eh}}+q_{\mathrm{h}} \dot{\rho}
\end{gathered}
$$

where $k_{\mathrm{e}}$ and $k_{\mathrm{h}}$ are the thermal conductivity of the electrons and heavy particles, respectively, $h_{\mathrm{e}}$ and $h_{\mathrm{h}}$ are the specific enthalpy of the electrons and heavy particles, respectively and $e$ is the electronic charge. $q_{\mathrm{e}}$ and $q_{\mathrm{h}}$ are the enthalpy transported with the mass flows. Other source terms are the elastic collision energy exchange term between the electrons and heavy species $Q_{\mathrm{eh}}$, the Joule heating $Q_{\mathrm{J}}$, and the radiation losses $Q_{\text {rad }}$. The last term on the right-hand side of equation (15) represents the electrons' heat conduction.

If the electron temperature is equal to the heavy particle temperature, equations (15) and (16) of the non-local thermodynamic equilibrium model (NLTE model) is reduced to a local thermodynamic equilibrium model (LTE model).

$$
\begin{aligned}
& \frac{\partial \rho h}{\partial t}+\nabla \cdot(\rho h \overrightarrow{\boldsymbol{u}})=\nabla \cdot\left(k_{\mathrm{T}} \nabla T\right)-Q_{\mathrm{rad}}+Q_{\mathrm{J}} \\
& \quad+\frac{5 k_{\mathrm{B}}}{2 e} \overrightarrow{\boldsymbol{J}} \cdot \nabla T+q_{T} \dot{\rho}
\end{aligned}
$$

where $k_{\mathrm{T}}=k_{\mathrm{e}}+k_{\mathrm{h}}, \quad h_{\mathrm{T}}=h_{\mathrm{e}}+h_{\mathrm{h}}$ and $q_{\mathrm{T}}=q_{\mathrm{e}}+q_{\mathrm{h}}$ are the total thermal conductivity and the total specific enthalpy and the enthalpy transported with the mass flow including contributions of both electrons and heavy particles, respectively.

Radiative transfer is an important energy transport mechanism in wall ablation controlled arcs. In most cases treating radiation from thermal plasmas, the NEC (net emission coefficient) method gives a good approximation of the radiation losses from the hottest regions and therefore is applied in our current work.

$$
Q_{\mathrm{rad}}=4 \pi \varepsilon_{r}
$$

where $\varepsilon_{r}$ is the net emission coefficient. The net emission coefficient for ablated PTFE vapor plasma is evaluated based on the work of Aubrecht and Bartlova [41].

The electron-heavy particle energy exchange term, which explicitly couples the heavy-particle and electron energy equations, is approximated by

$$
Q_{\mathrm{eh}}=\sum_{j} \frac{3}{2} k_{\mathrm{B}}\left(T_{\mathrm{e}}-T_{j}\right) n_{\mathrm{e}} \frac{2 m_{\mathrm{e}}}{m_{j}} \xi_{\mathrm{e} j}
$$

where $n_{\mathrm{e}}$ and $n_{j}$ are the electron and heavy particle number densities, and $m_{\mathrm{e}}$ and $m_{\mathrm{h}}$ are their respective masses. The volumetric collision frequency between the electrons and the heavy particles $\xi_{\text {eh }}$ is given by

$$
\xi_{\mathrm{e} j}=\left(\frac{8 k_{\mathrm{B}} T_{\mathrm{e}}}{m_{\mathrm{e}} \pi}\right)^{1 / 2} n_{j} \bar{Q}_{\mathrm{e} j}^{(1,1)}
$$

\section{(4) Electromagnetic equation}

The magnetic field is obtained by calculating the magnetic potential vector,

$$
\nabla \cdot\left(\nabla A_{i}\right)=-\mu j_{i}
$$

where $A_{i}, j_{i}$ and $\mu$ are the component of magnetic potential vector $\overrightarrow{\boldsymbol{A}}$, current density vector $\overrightarrow{\boldsymbol{J}}$ and the vacuum permeability, respectively.

The electrical field is determined using

$$
\overrightarrow{\boldsymbol{E}}=-\nabla \varphi
$$

where $\overrightarrow{\boldsymbol{E}}$ and $\varphi$ are the electric field vector and the electric potential. 
Table 1. Boundary conditions for the LTE and NLTE models.

\begin{tabular}{lllllll}
\hline Variables & $\mathrm{AB}$ & $\mathrm{BC}$ & $\mathrm{CD}$ & $\mathrm{AD}$ & $\mathrm{EF}$ & $\mathrm{DE}, \mathrm{CF}$ \\
\hline$P$ & $P_{\mathrm{n}}=0^{\mathrm{a}}$ & $P=P_{\text {sonic }} \mathrm{b}$ & $P_{\mathrm{n}}=0$ & $P_{\mathrm{n}}=0$ & $/$ & $/$ \\
$\overrightarrow{\boldsymbol{u}}$ & $u_{i, n}=0^{\mathrm{c}}$ & $u_{i, n}=u_{\text {sonic }}$ & $u_{i}=0$ & $u_{i}=0$ & $/$ & $/$ \\
$T$ & $T_{n}=0$ & $T_{n}=0$ & $T=T_{\text {wall }}$ & $T_{n}=0$ & $/$ & $/$ \\
$T_{\mathrm{h}}$ & $T_{\mathrm{h}, n}=0$ & $T_{h, n}=0$ & $T_{\mathrm{h}}=T_{\mathrm{wall}}$ & $T_{\mathrm{h}, n}=0$ & $/$ & $/$ \\
$T_{\mathrm{e}}$ & $T_{\mathrm{e}, n}=0$ & $T_{\mathrm{e}, n}=0$ & $T_{\mathrm{e}, n}=0$ & $T_{\mathrm{e}, n}=0$ & $/$ & $T_{\mathrm{m}}=300 \mathrm{~K}$ \\
$T_{\mathrm{m}}$ & $/$ & $/$ & $\phi_{q}$ & $/$ & $\varphi_{n}=0$ & $T_{\mathrm{m}, n}=0$ \\
$\varphi$ & $\varphi_{n}=0$ & $\varphi=0$ & Coupled & $-\sigma \varphi_{n}=J_{\text {anode }}$ & $A_{i}=0$ & $A_{i, n}=0$ \\
$\overrightarrow{\boldsymbol{A}}$ & $A_{i, n}=0$ & $A_{i, n}=0$ & Coupled & $A_{i, n}=0$ & \\
\hline
\end{tabular}

${ }^{\mathrm{a}} \mathrm{a}_{n}=\partial a / \partial n$ differentiation in the direction of the normal to the boundary.

${ }^{\mathrm{b}}$ Sonic boundary: the exit pressure is derived based on the expression of $P_{\text {sonic }}=u_{\text {sonic }}^{2} \rho / \gamma$ that the local velocity is equal to the local sound speed $u_{\text {sonic }}$.

${ }^{\mathrm{c}} i=r, x$ axisymmetric coordinates.

The current density is defined by

$$
\overrightarrow{\boldsymbol{J}}=\sigma \overrightarrow{\boldsymbol{E}}
$$

where $\sigma$ is electrical conductivity.

According to Maxwell's equations, we can evaluate the magnetic flux density $\overrightarrow{\boldsymbol{B}}$ from

$$
\overrightarrow{\boldsymbol{B}}=\nabla \times \overrightarrow{\boldsymbol{A}}
$$

(5) Equation of state

$$
\rho=\rho\left(P, T_{\mathrm{e}}, T_{\mathrm{h}}\right)
$$

3.2.2. Solid wall regions. The energy conservation equation is solved within the solid wall regions. The Joule heating effect is ignored as the electrical conductivity of the solid wall is very low, hence the equation becomes

$$
\frac{\partial \rho_{\mathrm{m}} c_{p, \mathrm{~m}} T_{\mathrm{m}}}{\partial t}-\nabla \cdot\left(k_{\mathrm{m}} \nabla T_{\mathrm{m}}\right)=0
$$

where $\rho_{\mathrm{m}}, c_{p, \mathrm{~m}}, k_{\mathrm{m}}$ and $T_{\mathrm{m}}$ are the mass density, the specific heat, the thermal conductivity and temperature of the solid wall, respectively.

\subsection{Boundary conditions}

Table 1 shows the boundary conditions used for the different variables in the LTE and NLTE models. Axisymmetric boundary conditions are imposed on the central axis $\mathrm{AB}$ : the radial velocity and the radial gradient of all dependent variables are zero. A sonic boundary condition is applied to the generator exit where the outlet pressure is set as $P_{\text {sonic }}=u_{\text {sonic }}^{2} \rho / \gamma$ which makes the local velocity along the central axis equal to the local sonic speed $u_{\text {sonic }}$. The annular cathode BC near the vent is set to be at zero electric potential. We impose a zero gradient boundary for the other transported variables.

The heavy particle temperature near the plasma boundary $\mathrm{CD}$ is equal to the temperature of the solid wall surface. A zero heat flux condition is defined for the electron energy equation. Additionally, the exchanges of mass, momentum and energy with the ablated vapor flow inwards are also accounted for by adding some source terms to the governing equations as described above. A heat flux density is imposed on the wall surface in order to solve the energy conservation equation in the solid wall [42].

$$
\phi_{q}=-\left.k_{\mathrm{m}} \frac{\partial T_{\mathrm{m}}}{\partial r}\right|_{r=R_{a}}=q(t)-\Delta H \cdot \Gamma-C_{p}\left(T_{\text {wall }}-T_{s}\right) \cdot \Gamma
$$

where $r=R_{a}$ corresponds to the inner dielectric surface, $\Delta H$ is the ablation heat, $\Gamma$ is the rate of polyethylene ablation per unit area, $T_{s}$ is the initial room temperature, $q(t)$ is the heat flux density, consisting of the radiative and particle convection fluxes determined by an expression similar to that used in [14] and $T_{\text {wall }}$ is the surface temperature. At the outer wall surface, a room temperature $T_{\mathrm{m}}=300 \mathrm{~K}$ is assumed. It should be noted, however, that the calculations show little influence of this value on the Teflon temperature distribution.

The anode spot is considered to be a circular area with a maximum radius of $1.5 \mathrm{~mm}$. The current density in the anode spot $\mathrm{AD}$ is represented by the following exponential function according to Hsu et al [43]:

$$
\begin{array}{ll}
J=J_{\max } \exp (-b r) & r \Leftarrow 1.5 \mathrm{~mm} \\
J=0 & r>1.5 \mathrm{~mm}
\end{array}
$$

where $J_{\max }=2.0 \times 10^{8} \mathrm{Am}^{-2}$. The coefficient $b$ is set according to the arc current $I$.

The interaction between the anode and the arc is not considered and an adiabatic wall (zero heat flux) has been used for its simplicity. Calculations show that the influence of this treatment on the arc solution is confined to a small region close to the anode.

\subsection{Property data}

Accurate data for the composition, thermodynamic properties and transport coefficients provide a prerequisite for a reliable numerical simulation of plasma behavior. For compressible flow and heat transfer simulations with ablated PTFE vapor as the plasma working gas, tabulated pressure-temperaturedependent thermodynamic and transport properties in wide pressure and temperature ranges were calculated based on the species composition taking into account 24 species, including atoms, ions and molecules as well as electrons as mentioned above [44]. To be more concrete, the thermodynamic properties were obtained from the internal energies 
employing standard thermodynamic relationships, also taking into account the Debye-Hückel correction as discussed by Kovitya [45]. Transport coefficients were calculated using the Chapman-Enskog method [46-49], which assumes that the species distribution function is a first-order perturbation to the Maxwellian distribution. The simplified approach of Devoto [50], in which the heavy-species and electron Boltzmann equations are decoupled, assuming that the change in the perturbation function in electrons is greater than that of the heavy species during heavy-species-electron interactions, was adopted. A third-order approximation was used, except for viscosity for which the second-order approximation was adopted [37, 38]. The cross-section data in the determination of the transport coefficients are from the compilation of the literature [51].

Figure 10 shows the contributions of the different species to the energy exchange term $Q_{\mathrm{eh}}$ in the electron temperature range up to $30000 \mathrm{~K}$ for non-equilibrium degrees of $\theta=1.2$ and 2. The importance of the carbon and fluorine atoms and ions contributions for electron temperatures higher than $8000 \mathrm{~K}$ is essentially caused by the fact that these species are the dominant species of the plasma for the temperature range mentioned above. For a higher non-equilibrium degree of $\theta=2$, the contribution of $\mathrm{CF}$ and $\mathrm{C}_{x}(x=2,3)$ neutral species for the energy exchange term through elastic collisions is also essentially important for temperatures lower than $8000 \mathrm{~K}$. This is because the decomposition of these molecular species is controlled by the heavy particle temperature which is lower with a larger non-equilibrium degree at a given electronic temperature. The ionization of atomic species does not happen until they are produced by the dissociation when the heavy particle temperature reaches the dissociation temperature. In our model, the energy exchange term as well as other plasma properties mentioned above are calculated and tabulated as a function of the electron temperature, plasma pressure and non-equilibrium degree.

\subsection{The wall-plasma interaction}

Following the same approach as Li et al's simulation [32], the plasma-wall interaction process is taken into account by implementing certain source terms in the governing equations. To be concrete, the radial transport of the corresponding mass, momentum and energy caused by the wall and plasma interaction is accounted for in the governing equations of one thin plasma element's layer adjacent to the capillary wall. It is noted that in the transition regime of the capillary operation both cases of ablation and deposition are possible and therefore we will consider both cases to account for the mass production or loss in the plasma bulk flow near the wall surface in the model. By means of this, a self-adjusting exchange mechanism during the iteration of the computer program can be formed [32]. The wall ablation rate is determined as described in section 2.1. The deposition rate can be determined as follows [42]:

$$
\Gamma=m_{2}\left(k T_{2} / m_{2}\right)^{0.5} n C_{2}
$$
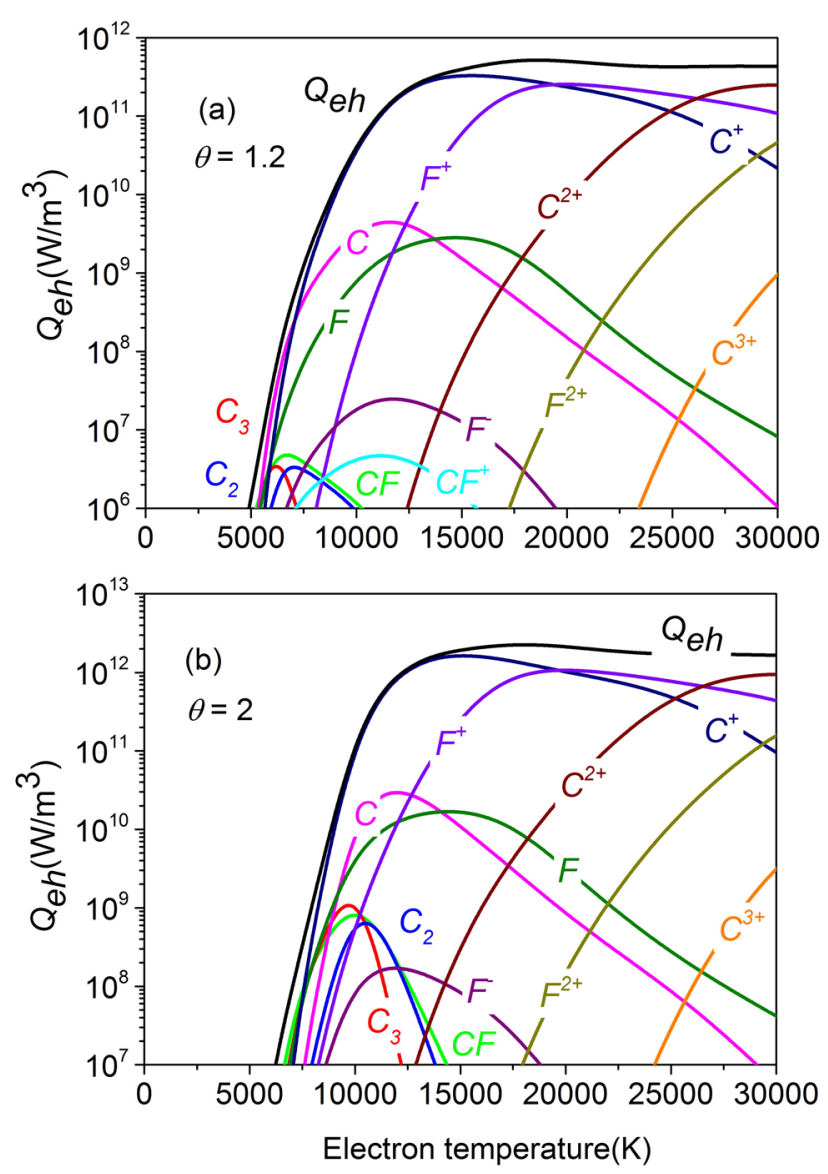

Figure 10. Contributions of the different species to the energy exchange term for the non-equilibrium degree of (a) $\theta=1.2$ and (b) $\theta=2$.

where $n C_{2}$ is the carbon particle density at the boundary (2), which is determined by the solution of the two-temperature plasma species model for PTFE vapor introduced in section 2.2. It is assumed that only the carbon molecules, atoms and ion depositions take place.

In the mass conservation equation, the volume mass generation or loss rate by ablation or deposition $\dot{\rho}$ is determined as follows:

$$
\dot{\rho}=\Gamma A_{\text {element }} / V_{\text {element }}
$$

where $A_{\text {element }}$ is the area of the solid wall surface element and $V_{\text {element }}$ is the volume of the plasma's element adjacent to the wall.

In the momentum conservation equation, the fifth term $v_{\text {flux }} \dot{\rho}$ represents the momentum exchange between the plasma and wall caused by ablation and deposition, and $v_{\text {flux }}$ is the velocity by which the mass enters or escapes the plasma element when ablation or deposition occurs. $v_{\text {flux }}$ is defined as the ratio of ablation or deposition rate to the plasma density $\Gamma / \rho$.

In the plasma energy conservation equation, the last term in equations (15)-(17) stands for the energy exchange between the plasma and wall caused by ablated or deposited mass flow, and $q$ is the energy transported with the mass flow.

$$
q_{i}=h_{i}\left(T_{2, \mathrm{e}}, T_{2, \mathrm{~h}}, P_{2}\right)+0.5 v_{\text {flux }}^{2} \quad i=e, h
$$



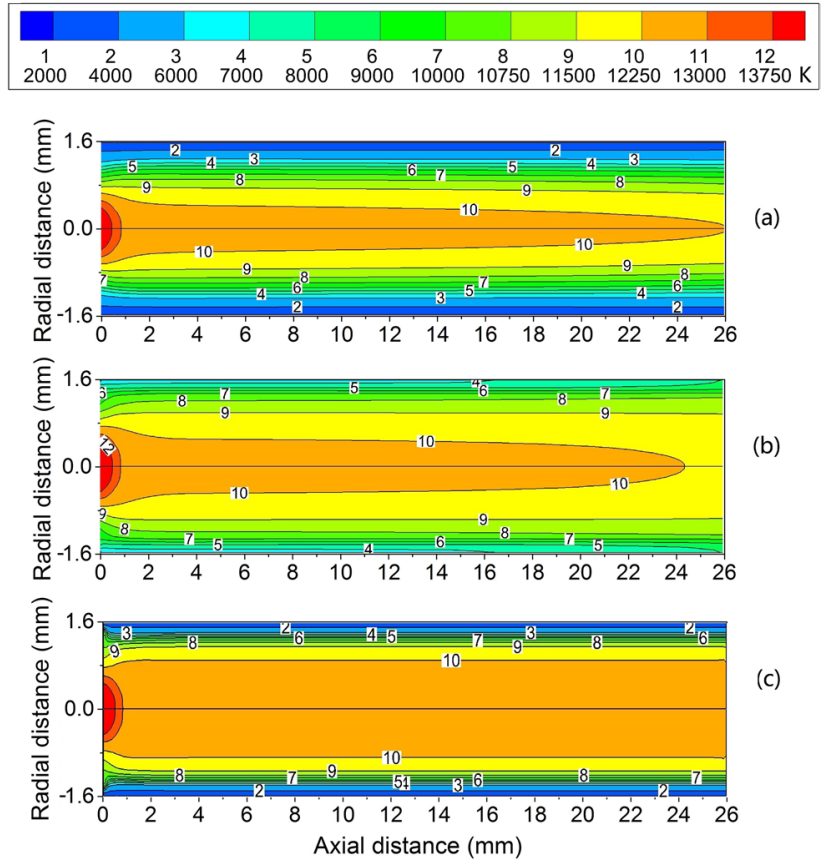

(b)

Figure 11. Steady temperature distribution for a current of $200 \mathrm{~A}$ : non-equilibrium electrons and heavy particle temperatures $T_{\mathrm{e}}$ (a) and $T_{\mathrm{h}}(\mathrm{b})$ as well as the equilibrium temperature $T$ (c).

where $h_{\mathrm{e}}$ and $h_{\mathrm{h}}$ are the electrons and heavy particles specific enthalpy in the plasma elements adjacent to the capillary wall.

\subsection{Modeling results and discussions}

3.6.1. Steady state calculations. Initial calculations were carried out for the steady-state case, and a comparison was performed between the predictions of LTE and NLTE calculations.

Figure 11 shows the steady temperature distribution of the plasma for a discharge current of 200 A. Both the temperatures of the electrons and heavy particles $T_{\mathrm{e}}$ and $T_{\mathrm{h}}$ obtained with the NLTE model as well as the equilibrium temperature $T$ with the LTE model are presented and compared with each other. The non-equilibrium heavy particle temperature is quite comparable to the electron temperature in the arc core. In the arc core, the electric energy obtained by electrons from the electric field can be effectively transferred to the heavy particles through elastic collisions because of the electron number density and hence the impact frequency between electrons and heavy particles is very high. However, the departure from equilibrium grows more rapidly when it comes towards the solid wall and the electron temperature is significantly larger than the heavy particle temperature at the wall surface. This is attributed to the influence of the plasma-cold wall interaction. The chemical reactions occurring in the wall ablation processes absorb the heat emitted from the arc and blocks most of the heat flux from the hot arc to further heat the wall, leaving a thin layer of relatively cold gas attached to the wall. As a result of the strong cooling effect of this cold boundary layer (cold wall), the plasma temperature decreases when it comes towards the wall. The electron number density and hence the collision frequency between electrons and heavy species

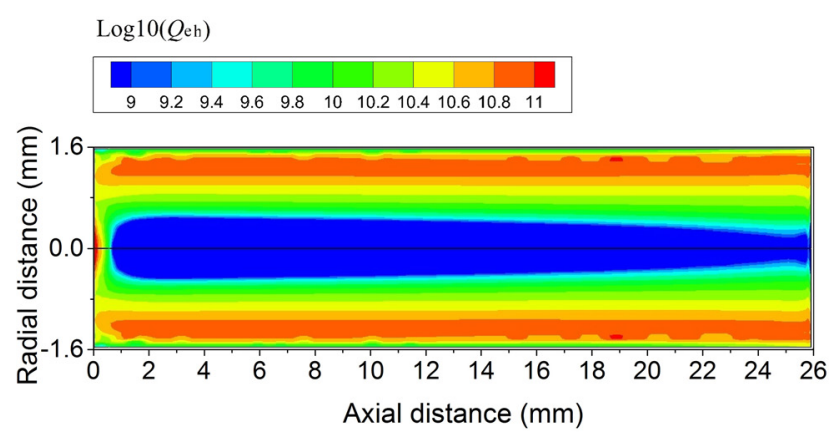

Figure 12. Steady distribution of energy exchange between the electrons and heavy particles for a current of 200 A calculated with the NLTE model.

decreases as well. The energy transfer between electrons and heavy particles, which links the electron and heavy species energy equations, is less effective and a departure from LTE takes place. This is also indicated in figure 12 where the 2D distribution of the energy exchange term between the electrons and heavy particles which couple the energy conservation equations for electrons and heavy particles are presented.

The elastic collision energy exchange grows progressively up to a maximum approximately $1.4 \mathrm{~mm}$ away from the central axis and then decreases towards the wall surface due to the decreasing plasma temperature. This trend is in agreement with the theoretical prediction for the wall stabilized $\mathrm{SF}_{6}$ arcs [52].

Although the plasma state near the central axis is close to thermal equilibrium, the equilibrium temperature we obtained with the LTE model is slightly higher than the $T_{\mathrm{e}}$ and $T_{\mathrm{h}}$ obtained with the NLTE model as presented in figure 13. This is due to the conductance nature, which is a volume integral. Indeed, from the radial distribution of the electrical conductivity at the axial location $x=13 \mathrm{~mm}$, which is also indicated in figure 13, the conducting radius predicted that the NLTE model is larger. Further, the gas temperature obtained with the LTE model shows an apparent discrepancy with that predicted with the NLTE model outside the arc core near the wall surface. This is partly due to the different boundary conditions used for the electrons energy equation (zero heat flux) and the heavy particles (gas) energy conservation equation (equal to the wall surface temperature). Moreover, the other dominant mechanism which can contribute to the deviation of the temperatures predicted with the LTE model and NLTE model is the occurrence of a significant thermal non-equilibrium region where the LTE model is not accurate enough to describe the plasma parameters. The non-equilibrium characteristics in the plasma elements near the wall surface indicate the need to consider the effect of the departure from LTE on the determination of the ablation rate.

Figure 14 shows the pressure field distribution predicted by both the LTE and NLTE model. The pressure is uniform in most of the capillary, but begins to decrease steeply near the vent, falling to its lowest level at the exit. This trend is in good agreement with the previously-presented simulation using the LTE model for PE ablation by Li et al [32]. We further note that the maximum pressure calculated with the LTE model is larger than that with the NLTE model. This is attributed to the overestimated temperature with the LTE model at $200 \mathrm{~A}$ as 


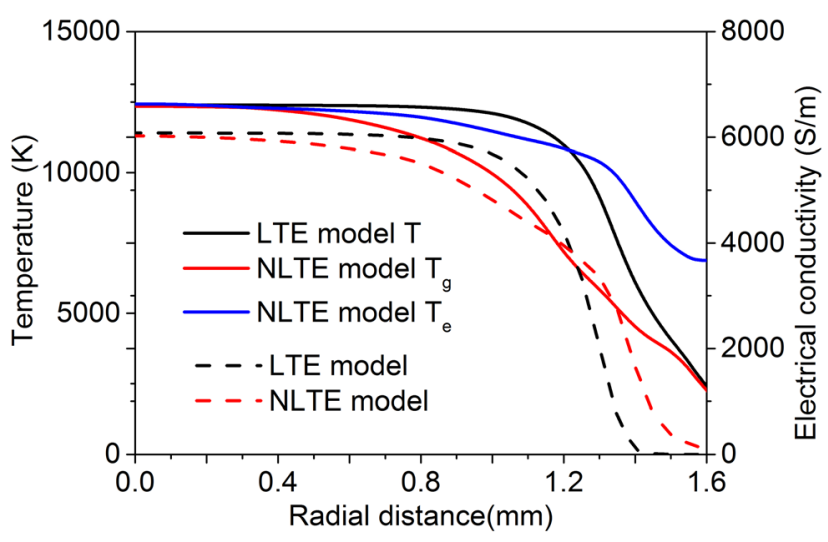

Figure 13. Radial distribution of plasma temperature (solid line) and electrical conductivity (dashed line) at $200 \mathrm{~A}$ at the location $x=13 \mathrm{~mm}$.
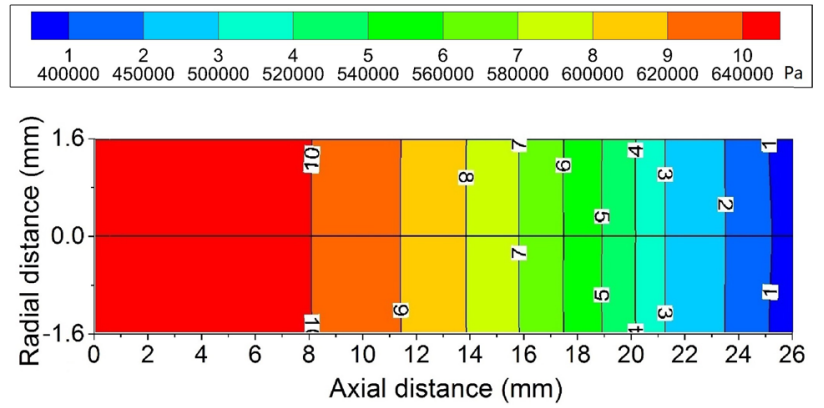

(a)
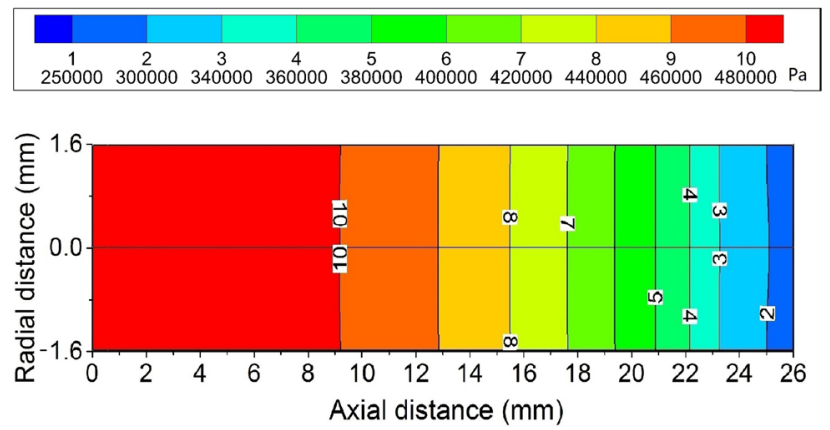

(b)

Figure 14. Steady distribution of the pressure predicted with the LTE model (a) and NLTE model (b) for a 200 A current.

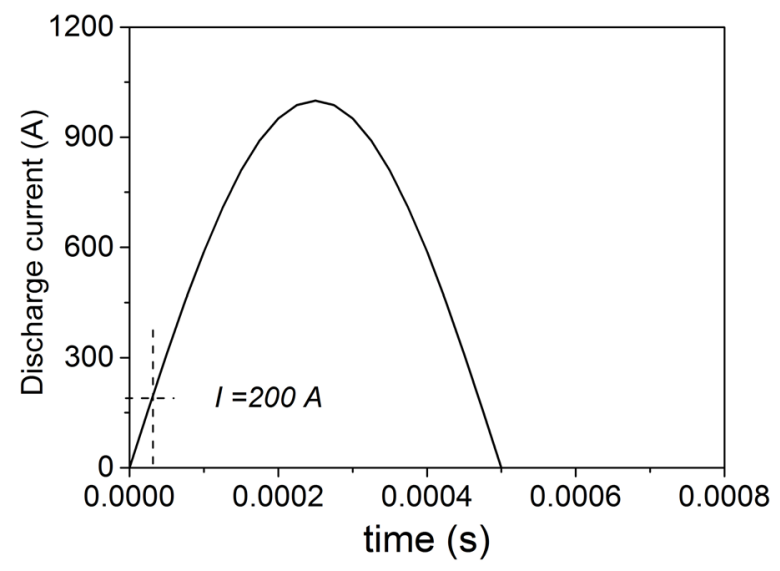

Figure 15. Current profile used in our model.
Temperature (kK)

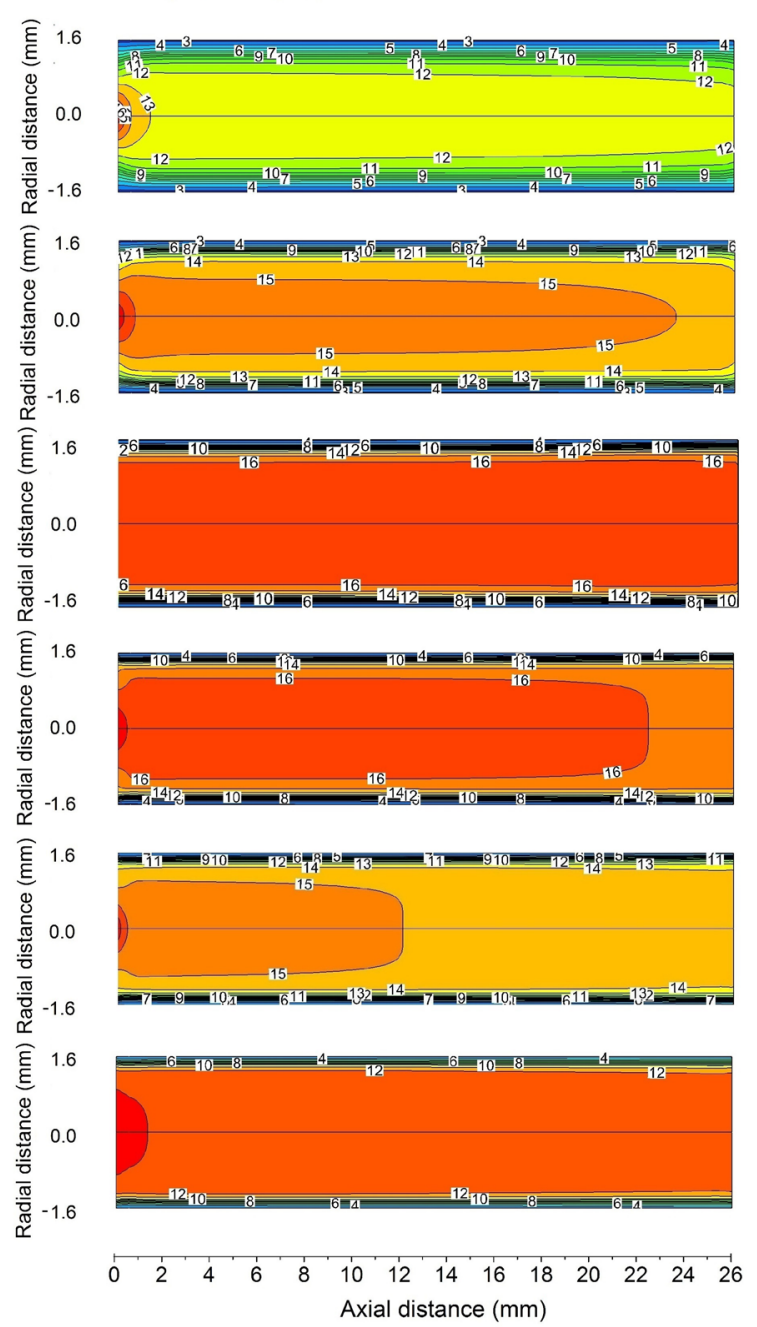

(a)

Figure 16. Contours of gas (heavy particle) temperature predicted with the NLTE model in the capillary at different times (a) $45 \mu \mathrm{s}$, (b) $125 \mu \mathrm{s}$, (c) $250 \mu \mathrm{s}$, (d) $375 \mu \mathrm{s}$, (e) $450 \mu \mathrm{s}$, and (f) $600 \mu \mathrm{s}$.

discussed above. The wall surface temperature calculated with the LTE model is also overestimated. For example, a wall surface temperature of $903 \mathrm{~K}$ at the location $x=13 \mathrm{~mm}$ in the LTE case is reached compared to $901 \mathrm{~K}$ with the NLTE model. This leads to an overestimated ablation rate $1.32 \times 10^{-3} \mathrm{~kg} \mathrm{~s}^{-1}$. In contrast, the NLTE model gives a smaller ablation rate of $1.15 \times 10^{-3} \mathrm{~kg} \mathrm{~s}^{-1}$.

3.6.2. Transient state calculations. In this paper, we use the sin wave discharge current presented in figure 15 to investigate the characteristics of the capillary. In our simulation, the transient calculation starts from an initial steady state of $200 \mathrm{~A}$ at $32 \mu \mathrm{s}$ in order to avoid the arc initiation phenomena.

Figures 16-18 show the contours of gas temperature, pressure and speed, respectively predicted with the NLTE model in the capillary at different times. One can see that the gas temperature temporal distribution is closely linked with the discharge current value. With the increase in the discharge current, the rising plasma temperature becomes more concentrated and shows a relatively even radial distribution within the hottest arc core. In contrast, a large gradient of gas 


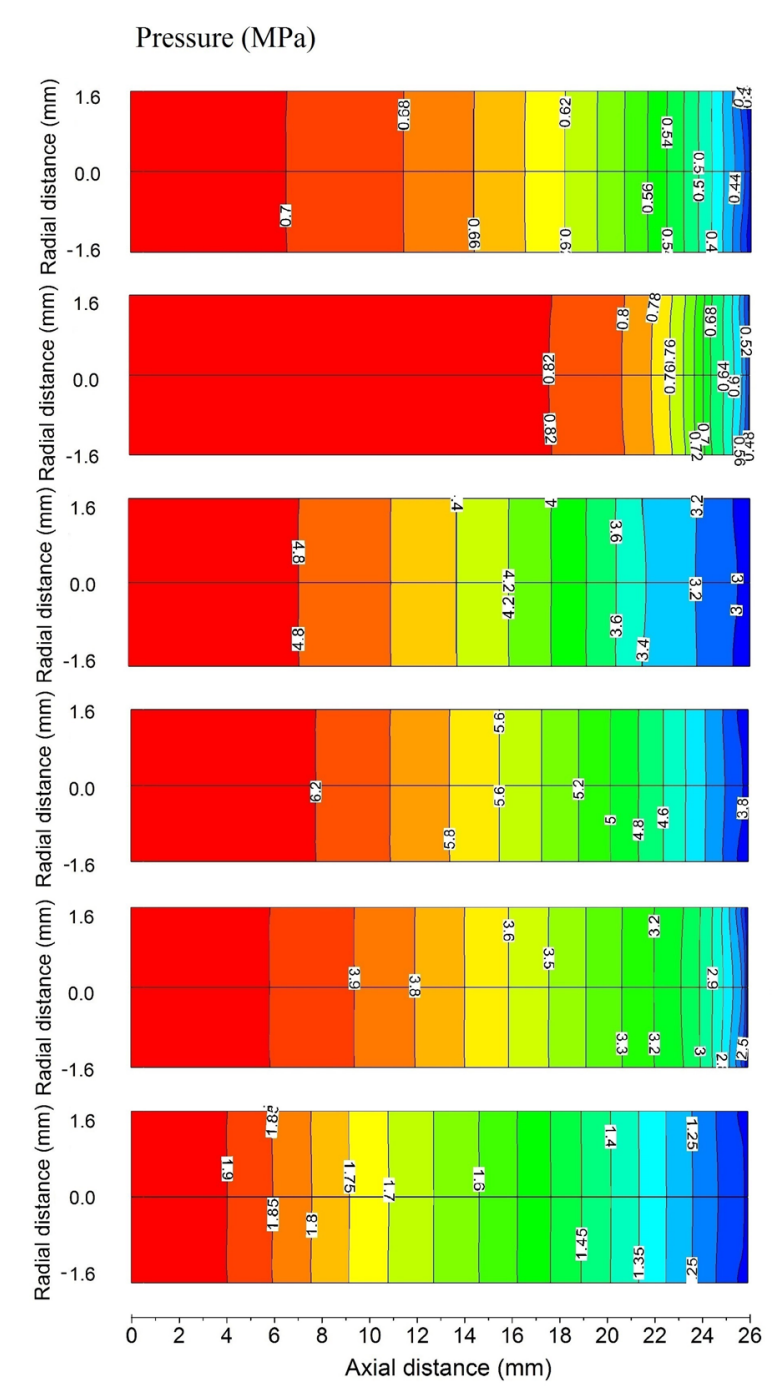

Figure 17. Contours of plasma pressure predicted with the NLTE model in the capillary at different times (a) $45 \mu \mathrm{s}$, (b) $125 \mu \mathrm{s}$, (c) $250 \mu \mathrm{s}$, (d) $375 \mu \mathrm{s}$, (e) $450 \mu \mathrm{s}$, and (f) $600 \mu \mathrm{s}$.

temperature exists in the thin layer near the wall surface as a result of the strong cooling effect by the cold wall. One can see that the gas temperature still has a very high value regardless of the decay of the discharge current from $t=250 \mu \mathrm{s}$. The peak gas temperature at $t=375 \mu$ s is even higher than that at $t=250 \mu$ s showing that the temperature wave delays compared with the current wave. This is because the temperature cannot adjust to arrive at the steady equilibrium state in a short period to respond to the very drastic change of the discharge current. After the discharge current reaches zero at $t=500$ $\mu \mathrm{s}$, the gas temperature in the arc core gradually decreases to around $12000 \mathrm{~K}$ at $t=600 \mu \mathrm{s}$.

Figure 17 shows that the largest pressure increases with the discharge current before the latter peaks at $t=250 \mu$ s and still increases to a higher value of $6.2 \mathrm{MPa}$ at $t=375 \mu \mathrm{s}$. With the further decrease of the discharge current, the pressure decreases towards the end of the pulse to about 3.2 MPa. The gas velocity increases towards the capillary and reaches the maximum value at the exit. It is noted that the delay of the plasma temperature and the pressure near the exit compared with the current wave is relatively smaller than that in the upstream area when we
Velocity $(\mathrm{km} / \mathrm{s})$

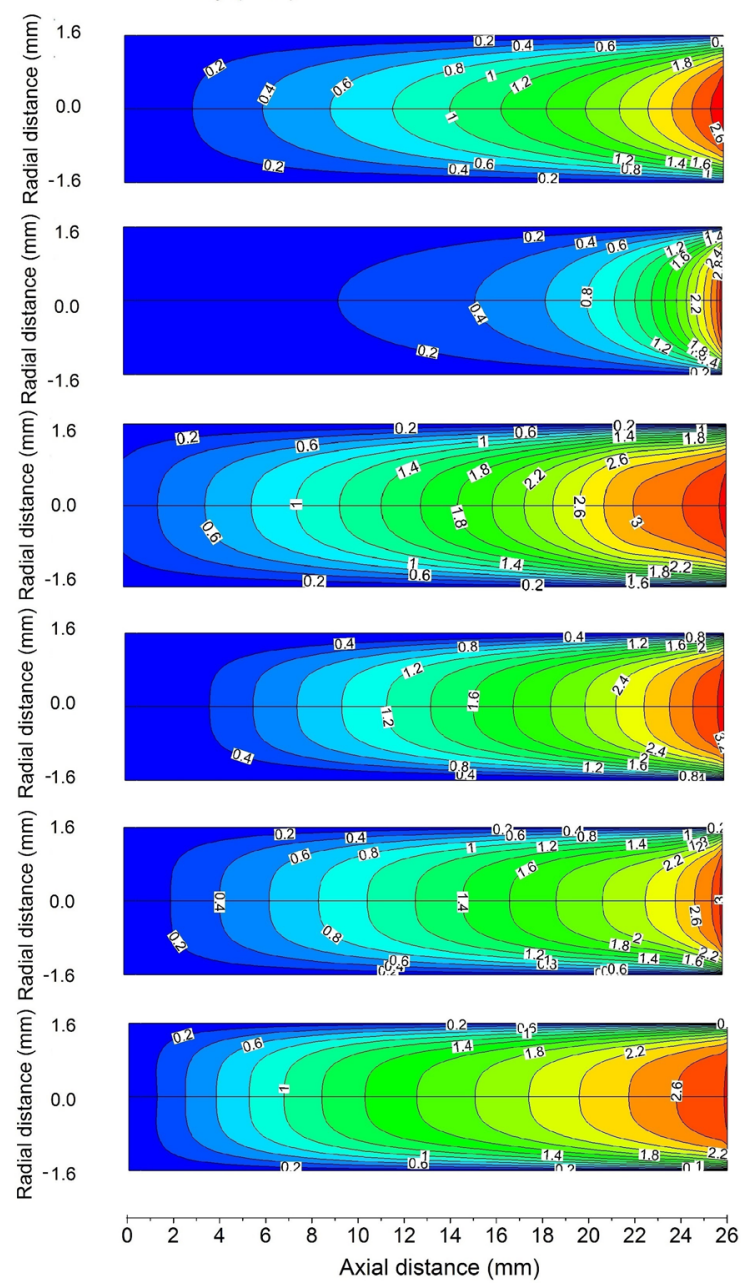

Figure 18. Contours of velocity predicted with the NLTE model in the capillary at different times (a) $45 \mu \mathrm{s}$, (b) $125 \mu \mathrm{s}$, (c) $250 \mu \mathrm{s}$, (d) $375 \mu \mathrm{s}$, (e) $450 \mu \mathrm{s}$, and (f) $600 \mu \mathrm{s}$.

check their distributions in figures 16 and 17, respectively. As a result, the local sonic velocity (also the peak velocity) in the capillary generally follows the transient evolution of current.

In figure 19, we present the evolution of the integrated net ablation rate of the whole capillary as well as the wall surface temperature predicted by both the NLTE model and LTE model. Only a slight variation in the surface temperature along the capillary is found, here we only present the results at the axial location $x=13 \mathrm{~mm}$. Similar to the pressure and plasma temperature, the waves of the integrated ablation rate and the wall surface temperature are delayed compared with the current wave. Moreover, it is noted that the wall surface temperature and hence the integrated ablation rate is still very high after the pulse. This post-pulse wall ablation will increase the total mass ablation.

With the increase in current, the surface temperature and the ablation rate generally show a rising trend. However, we can see that the integrated ablation rate curve shows several fluctuations. This is closely linked to the competition between the rise in speed of the equilibrium vapor pressure (determined by the surface temperature) and the pressure in the capillary which also increases with the current [53]. As indicated in figure 8, at 


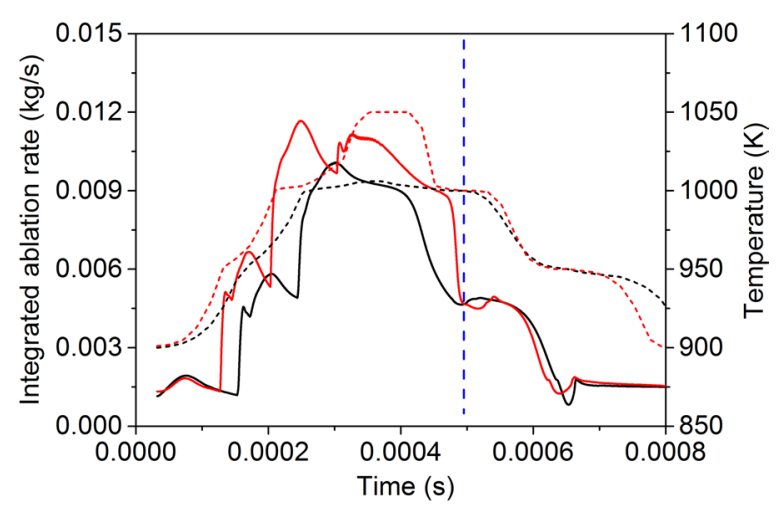

Figure 19. Integrated net ablation rate (solid line) and wall surface temperature (dashed line) at $x=13 \mathrm{~mm}$ versus transient time. Black lines: NLTE model; red lines: LTE model. The blue dashed line corresponds to the time instant $t=500 \mu$ s when the current decreases to zero.

a fixed wall surface temperature and plasma temperature, with the increase in the pressure, the ablation rate firstly increases and then decreases when the pressure rises towards the critical value, i.e. the equilibrium vapor pressure. With the increase in the discharge current, when the plasma pressure at certain axial positions exceeds the equilibrium vapor pressure, the ablation rate reaches zero and the deposition occurs instead of ablation. Similarly, with the increase in the discharge current, both the wall surface temperature and the plasma temperature increase. As we can see from figure 8, at a fixed plasma pressure, the ablation rate decreases and increases, respectively, with the increase in the plasma temperature and the wall surface temperature. Therefore, whether the ablation rate increases or not also depends on the result of the competition between the rise in speed of the plasma temperature and the wall surface temperature with the increasing current. This can also contribute to the transient fluctuation of the ablation rate. When the wall surface temperature reaches its peak and stays relatively stable, the increase in the plasma temperature leads to a reduction in the ablation rate. Of these two above mentioned factors, the competition between the rise in the speed of the equilibrium vapor pressure contributes most to the fluctuated ablation rate as a result of a much more drastic transient change of the pressure compared with the change of the plasma temperature.

Compared with the results predicted with the NLTE model, the LTE model overestimates the wall surface temperature and hence the ablation rate on average. This is attributed to a different two-temperature profiles predicted with the NLTE model especially in the near wall layer where departure from equilibrium prevails as discussed in the steady case. Over the whole discharge period of $0.8 \mathrm{~ms}$, the cumulative ablated mass predicted with the NLTE model is $3.60 \mathrm{mg}$ which is $22 \%$ lower than that predicted with the LTE model $(4.35 \mathrm{mg})$.

\section{Conclusions}

The LTE approximation is often violated in the plasma bulk flow near the wall surface due to the strong cooling effect of the solid wall. Therefore, this paper modified the widely used two-layer kinetic ablation model and for the first time, applied it to an ablation controlled non-equilibrium discharge. A twotemperature plasma model considering the possible departure from thermal equilibrium is also developed for the first time to describe the plasma bulk of abated PTFE vapor adjacent to the solid wall. The plasma species composition as well as the physical parameters (average particle mass and specific heat ratio), which are required to acquire the ablation rate, are determined as a function of electron temperature, pressure and nonequilibrium degree. Ablation data of PTFE calculated with the improved two-layer kinetic ablation model was presented and applied to a two-temperature MHD model simulation of an ablation-dominated discharge for an ETC launch device. Additionally, for the first time, the effect of the time dependent wall surface temperature on the ablation rate and plasma parameters has been investigated in a $2 \mathrm{D}$ geometry by a selfconsistent calculation of the wall temperature distribution.

During the solution of the MHD model, the transport coefficients and thermodynamic properties as well as the energy exchange term, which are necessary for the numerical solution of the MHD model equations, are calculated. The exchange of mass, momentum and energy between non-equilibrium plasma and the solid propellant surface associated with the ablated vapor influx were carefully treated. Further, both the ablation and the deposition process are taken into account. A detailed comparison of the results of the non-equilibrium model with those of an equilibrium model is presented to investigate the influence of the departure from the thermal equilibrium on the ablation characteristics and the field distribution of plasma properties. The following conclusions can be drawn from this paper:

(1) Departure from thermal equilibrium can change the plasma species composition and hence the plasma parameters such as the mass density as well as the average particle mass. Therefore, the calculated ablation rate is sensitive to the non-equilibrium effect which occurs in the plasma element near the ablated wall surface. Our improved twolayer kinetic model which takes into account the possible departure from equilibriums in the plasma is preferable in the determination of the ablation rate.

(2) Our assessment of the property distributions of an ablationdominated discharge for an ETC launch device by means of a two-temperature MHD model has shown that the departure from thermal equilibrium takes place in the plasma element adjacent to the wall surface. The deviation of the plasma parameters and the ablation rate as well as the cumulative ablated mass over the whole discharge period predicted with the NLTE model and LTE model indicates the need for the consideration of the influence of the departure from LTE on the determination of ablation rate.

(3) Our calculation shows that the waves of plasma properties and wall temperature is delayed compared with the current wave. This is because the discharge current changes very rapidly, the system cannot adjust to arrive at the steady equilibrium state in a short period. Especially, the surface temperature still poses a very high value after the discharge pulse. This leads to a post-pulse wall ablation and increases the total mass ablation. 


\section{Acknowledgments}

This work was supported by National Natural Science Foundation of China (Grant Nos. 51407182, 11675040), Beijing Natural Science Foundation (Grant No. 3154044), the open project of State Key Laboratory of Electrical Insulation and Power Equipment (Grant No. EIPE14208), along with Innovative Research Fund and Internship Project of Qian Xuesen Laboratory of Space Technology. The authors would like to acknowledge Dr Rui Li for his kind help and valuable discussions.

\section{References}

[1] Zhang J L, Yan J D, Murphy A B, Hall W and Fang M T C 2002 Computational investigation of arc behavior in an auto-expansion circuit breaker contaminated by ablated nozzle vapor IEEE Trans. Plasma Sci. 2 706-19

[2] Yang F, Wu Y, Rong M Z and Niu C P 2013 Low-voltage circuit breaker arcs-simulation and measurements J. Phys. D: Appl. Phys. 46273001

[3] Paul K C, Sakuta T, Takashima T and Ishikawa M 1997 The dynamic behaviour of wall-stabilized arcs contaminated by Cu and PTFE vapours J. Phys. D: Appl. Phys. 30103

[4] Hong D, Dussart R, Cachoncinlle C, Rosenfeld W, Gotze S, Pons J, Viladrosa R, Fleurier C and Pouvesle J M 2000 Study of a fast ablative capillary discharge dedicated to soft x-ray production Rev. Sci. Instrum. 71 15-9

[5] Schönherr T, Nees F, Arakawa Y, Komurasaki K and Herdrich G 2013 Characteristics of plasma properties in an ablative pulsed plasma thruster Phys. Plasmas 20033503

[6] Keidar M, Boyd I D and Beilis I I 2000 Electrical discharge in the Teflon cavity of a coaxial pulsed plasma thruster IEEE Trans. Plasma Sci. 28 376-85

[7] Burton R L and Turchi P J 1998 Pulsed plasma thruster J. Propul. Power 14 716-35

[8] Kim S H, Yang K S, Lee Y H, Kim J S and Lee B H 2009 Electrothermal-chemical ignition research on $120 \mathrm{~mm}$ gun in Korea IEEE Trans. Magn. 45 341-6

[9] Keidar M and Boyd I D 2006 Ablation study in the capillary discharge of an electrothermal gun J. Appl. Phys. 99053301

[10] Li X W, Li R, Jia S L and Zhang Y C 2012 Interaction features of different propellants under plasma impingement J. Appl. Phys. 12063303

[11] Autrique D, Gornushkin I, Alexiades V, Chen Z, Bogaerts A and Rethfeld B 2013 Revisiting the interplay between ablation, collisional and radiative processes during ns-laser ablation Appl. Phys. Lett. 103174102

[12] Wang Z B, Hong M H, Lu Y F, Wu D J, Lan B and Chong T C 2003 Femtosecond laser ablation of polytetrafluoroethylene (Teflon) in ambient air J. Appl. Phys. 936375

[13] Langmuir I 1913 The vapor pressure of metallic tungsten Phys. Rev. 2 329-42

[14] Keidar M, Boyd I D and Beilis I 2000 Electrical discharge in the Teflon cavity of a coaxial pulsed plasma thruster IEEE Trans. Plasma Sci. 28 376-85

[15] Powell J D and Zielinski A E 1993 Capillary discharge in the electrothermal gun IEEE Trans. Magn. 29 591-6

[16] Raja L L, Varghese P K and Wilson D E 1997 Modeling of the electrogun metal vapor plasma discharge $J$. Thermophys. Heat Transf. 11 353-60

[17] Zaghloul M R, Bourham M A and Doster J M 2001 Semianalytical modelling and simulation of the evolution and flow of Ohmically-heated non-ideal plasmas in electrothermal guns J. Phys. D: Appl. Phys. 34 772-86
[18] Kim K 2005 Numerical simulation of capillary plasma flow generated by high-current pulsed power Int. J. Therm. Sci. 44 1039-46

[19] Christen T 2007 A maximum entropy production model for Teflon ablation by arc radiation J. Phys. D: Appl. Phys. 405719

[20] Keidar M, Boyd I D and Beilis I I 2001 On the model of Teflon ablation in an ablation-controlled discharge J. Phys. D: Appl. Phys. 34 1675-7

[21] Porwitzky A J, Keidar M and Boyd I D 2009 Numerical parametric study of the capillary plasma source for electrothermal-chemical guns IEEE Trans. Magn. 45 574-7

[22] Pekker L, Keidar M and Cambier J L 2008 Effect of thermal conductivity on the Knudsen layer at ablative surfaces J. Appl. Phys. 103906

[23] Keidar M, Boyd I D, Williams A and Beyer R 2007 Ablation study in a capillary sustained discharge IEEE Trans. Magn. 43 308-12

[24] Keidar M and Beilis I I 2006 Nonequilibrium thermal boundary layer in a capillary discharge with an ablative wall Phys. Plasmas 13114503

[25] Zaghloul M R 2004 On the vaporization of Teflon and heated compound-materials in ablation-controlled arcs J. Appl. Phys. 953339

[26] Zaghloul M R 2008 Improved modelling of electrothermal plasma source with radiation transport J. Phys. D: Appl. Phys. 41225206

[27] Li R, Li X W, Jia S L, Murphy A B and Shi Z Q 2010 Comparisons of kinetic ablation models for the capillary discharge J. Appl. Phys. 108023301

[28] Yang L, Liu X Y, Zhiwen Wu and Wang N F 2014 Study on the wall ablation of heated compound-materials plasmas based on a modified model Appl. Phys. Lett. 104084102

[29] Li R, Li X W, Jia S L, Murphy A B and Shi Z Q 2010 Study of different models of the wall ablation process in capillary discharge IEEE Trans. Plasma Sci. 381033

[30] Anisimov S I 1968 Vaporization of metal absorbing laser radiation Sov. Phys.-JETP 27 182-3

[31] Knight C J 1979 Theoretical modeling of rapid surface vaporization with back pressure AIAA J. 17 519-23

[32] Li R, Li X W, Jia S L, Murphy A B and Shi Z Q 2011 A two-dimensional capillary discharge model considering the ablation and deposition processes J. Appl. Phys. 110093302

[33] Keidar M and Beilis I 2013 Plasma Engineering: Applications from Aerospace to Bio and Nanotechnology (New York: Academic)

[34] Rat V, Murphy A B, Aubreton J, Elchinger M F and Fauchais P 2008 Treatment of non-equilibrium phenomena in thermal plasma flows J. Phys. D: Appl. Phys. 41183001

[35] Trelles J P, Heberlein J V R and Pfender E 2007 Nonequilibrium modeling of arc plasma torches $J$. Phys. D: Appl. Phys. 40 5937-52

[36] Wang W Z, Yan J D, Rong M Z and Spencer J W 2013 Theoretical investigation of the decay of an $\mathrm{SF}_{6}$ gasblast arc using a two-temperature hydrodynamic model J. Phys. D: Appl. Phys. 46065203

[37] Wang W Z, Rong M Z, Wu Y, Spencer J W, Yan J D and Mei D H 2012 Thermodynamic and transport properties of two-temperature $\mathrm{SF}_{6}$ plasmas Phys. Plasmas 19083506

[38] Wang W Z, Rong M Z, Yan J D, Murphy A B and Spencer J W 2011 Thermophysical properties of nitrogen plasmas under thermal equilibrium and non-equilibrium conditions Phys. Plasmas 18113502

[39] van de Sanden M C M, Schram P P J M, Peeters A G, van der Mullen J A M and Kroesen G M W 1989 Thermodynamic generalization of the Saha equation for a two-temperature plasma Phys. Rev. A 40 5273-6 
[40] Li J Q, Litzinger T A, Das M and Thynell S T 2006 Study of plasma-propellant interaction during normal impingement J. Propul. Power 22 929-37

[41] Aubrecht V and Bartlova M 2009 Net emission coefficients of radiation in air and $\mathrm{SF}_{6}$ thermal plasmas Plasma Chem. Plasma Process. 29 131-47

[42] Keidar M and Boyd I D 2006 Ablation study in the capillary discharge of an electrothermal gun J. Appl. Phys. 99053301

[43] Hsu K C, Etemadi K and Pfender E 1983 Study of the free-burning high-intensity argon arc J. Appl. Phys. 54 1293-301

[44] Wang W Z, Kong L H, Geng J Y, Wei F Z and Xia G Q Two temperature plasma properties of heated Teflon vapour for ablation controlled discharge applications J. Phys. D: Appl. Phys. in preparation

[45] Kovitya P 1985 Physical properties of high-pressure plasmas of hydrogen and copper in the temperature range 5000-60000 K IEEE Trans. Plasma Sci. 13 587-94

[46] Hirschfelder J O, Curtis C F and Bird R B 1964 Molecular Theory of Gases and Liquids 2nd edn (New York: Wiley)
[47] Chapman S and Cowling T G 1970 The Mathematical Theory of Non-uniform Gases 3rd edn (Cambridge: Cambridge University Press)

[48] Ferziger J H and Kaper H G 1972 Mathematical Theory of Transport Processes in Gases (North-Holland: Amsterdam)

[49] Rat V, André P, Aubreton J, Elchinger M F, Fauchais P and Lefort A 2002 Transport coefficients including diffusion in a two-temperature argon plasma J. Phys. D: Appl. Phys. 35 981-91

[50] Devoto R S 1973 Transport coefficients of ionized argon Phys. Fluids 16 616-23

[51] Wang W Z, Wu Y, Rong M Z, Éhn L and Černušák I 2012 Thermodynamic properties and transport coefficients of $\mathrm{F}_{2}$, $\mathrm{CF}_{4}, \mathrm{C}_{2} \mathrm{~F}_{2}, \mathrm{C}_{2} \mathrm{~F}_{4}, \mathrm{C}_{2} \mathrm{~F}_{6}, \mathrm{C}_{3} \mathrm{~F}_{6}$ and $\mathrm{C}_{3} \mathrm{~F}_{8}$ plasmas J. Phys. D: Appl. Phys. 45285201

[52] Girard R, Gonzalez J J and Gleizes A 1999 Modelling of a two-temperature $\mathrm{SF}_{6}$ arc plasma during extinction J. Phys. D: Appl. Phys. 32 1229-38

[53] Hang Y H, Li X W, Wu J, Zhao W Y, Li R and Jia S L 2016 Study of a 2D time-dependent capillary discharge model IEEE Trans. Plasma Sci. 4 715-21 